\title{
Higher spin black holes with soft hair
}

\section{Daniel Grumiller, ${ }^{a}$ Alfredo Pérez, ${ }^{b}$ Stefan Prohazka, ${ }^{a}$ David Tempo $^{b}$ and Ricardo Troncoso $^{b}$}

\author{
${ }^{a}$ Institute for Theoretical Physics, TU Wien, \\ Wiedner Hauptstrasse 8-10/136, Vienna, A-1040 Austria \\ ${ }^{b}$ Centro de Estudios Cientificos (CECs), \\ Av. Arturo Prat 514, Valdivia, Chile \\ E-mail: grumil@hep.itp.tuwien.ac.at, aperez@cecs.cl, \\ prohazka@hep.itp.tuwien.ac.at, tempo@cecs.cl, troncoso@cecs.cl
}

ABSTRACT: We construct a new set of boundary conditions for higher spin gravity, inspired by a recent "soft Heisenberg hair"-proposal for General Relativity on three-dimensional Anti-de Sitter space. The asymptotic symmetry algebra consists of a set of affine $\hat{u}(1)$ current algebras. Its associated canonical charges generate higher spin soft hair. We focus first on the spin- 3 case and then extend some of our main results to spin- $N$, many of which resemble the spin-2 results: the generators of the asymptotic $W_{3}$ algebra naturally emerge from composite operators of the $\hat{u}(1)$ charges through a twisted Sugawara construction; our boundary conditions ensure regularity of the Euclidean solutions space independently of the values of the charges; solutions, which we call "higher spin black flowers", are stationary but not necessarily spherically symmetric. Finally, we derive the entropy of higher spin black flowers, and find that for the branch that is continuously connected to the BTZ black hole, it depends only on the affine purely gravitational zero modes. Using our map to $W$-algebra currents we recover well-known expressions for higher spin entropy. We also address higher spin black flowers in the metric formalism and achieve full consistency with previous results.

KEYwords: Black Holes, Classical Theories of Gravity, Gauge-gravity correspondence

ARXIV EPRINT: 1607.05360 


\section{Contents}

1 Introduction 1

2 Asymptotic structure 2

2.1 Asymptotic symmetries and the algebra of the canonical generators 3

2.2 (Higher spin) soft hair 4

3 Highest weight gauge and the emergence of composite $W_{3}$ symmetries 5

4 Higher spin black holes with soft hair $\quad 8$

4.1 Regularity and black hole entropy in diagonal gauge 8

$\begin{array}{lll}4.2 & \text { Remarks on regularity and entropy in highest weight gauge } & 10\end{array}$

5 Higher spin black holes with soft hair in the metric formalism 12

6 Extension to $\mathrm{AdS}_{3}$ gravity coupled to fields of spin greater than $2 \quad 14$

$\begin{array}{lll}7 & \text { Discussion } & 16\end{array}$

A Principal embedding of SL $(2, \mathbb{R})$ within SL $(N, \mathbb{R}) \quad 17$

$\begin{array}{ll}\text { B Explicit form of the spin-3 field } & 18\end{array}$

\section{Introduction}

It has recently been shown that General Relativity with negative cosmological constant in three spacetime dimensions admits a new class of stationary black holes that are not necessarily spherically symmetric and do not fulfill the Brown-Henneaux boundary conditions [1]. A consistent set of boundary conditions that accommodates this class of solutions was then constructed, from which it can be seen that the generic black flower configuration is endowed with an infinite number of affine $\hat{u}(1)$ charges that commute with the total Hamiltonian. These charges then correspond to soft hair in the sense of Hawking, Perry and Strominger [2]. It was also shown in [1] that the precise levels of the $\hat{u}(1)$ generators are such that the Virasoro algebra with the Brown-Henneaux central extension [3] naturally emerges as a composite one through a twisted Sugawara construction. An additional remarkable feature of the boundary conditions aforementioned is that the spectrum, given by the space of solutions that fulfill them, is such that regularity of the configurations at the horizon holds independently of the global charges.

One of our main goals is to extend these results to the case of gravity on $\mathrm{AdS}_{3}$ coupled to bosonic higher spin fields [4-6]. In this case, it is known that the infinite tower of fields can 
be consistently truncated so as to describe a finite number of interacting nonpropagating fields of spin $s=2,3, \cdots, N$, see e.g. [7, 8]. The theory can then be generically described in terms of the difference of two Chern-Simons actions for independent gauge fields $A^{ \pm}$ that take values in $s l(N, \mathbb{R})$, so that the action reads

$$
I=I_{\mathrm{CS}}\left[A^{+}\right]-I_{\mathrm{CS}}\left[A^{-}\right],
$$

with

$$
I_{\mathrm{CS}}[A]=\frac{k_{N}}{4 \pi} \int \operatorname{tr}\left(A \wedge d A+\frac{2}{3} A \wedge A \wedge A\right),
$$

where $\operatorname{tr}(\cdots)$ stands for the trace in the fundamental representation of $\operatorname{sl}(N, \mathbb{R})$ (see appendix A). The level in (1.2) relates to the Newton constant and the AdS radius according to $k_{N}=\frac{k}{2 \epsilon_{N}}=\frac{\ell}{8 G \epsilon_{N}}$, whose normalization is determined by $\epsilon_{N}=\frac{N\left(N^{2}-1\right)}{12}$.

The gauge fields are related to a suitable generalization of the dreibein and the spin connection, defined through

$$
A^{ \pm}=\omega \pm \frac{e}{\ell}
$$

and hence, the spacetime metric and the higher spin fields can be reconstructed from

$$
g_{\mu \nu}=\frac{1}{\epsilon_{N}} \operatorname{tr}\left(e_{\mu} e_{\nu}\right) \quad \Phi_{\mu_{1} \ldots \mu_{s}}=\frac{1}{\epsilon_{N}^{(s)}} \operatorname{tr}\left(e_{\left(\mu_{1} \ldots e_{\left.\mu_{s}\right)}\right)}\right.
$$

being manifestly invariant under an extension of the local Lorentz group described by the diagonal subgroup of $\mathrm{SL}(N, \mathbb{R}) \otimes \mathrm{SL}(N, \mathbb{R})$. Diffeomorphisms and higher spin transformations are then related to the remaining gauge symmetries. The parentheses around the subscripts denote symmetrization and the spin-3 field in our $\operatorname{sl}(3, \mathbb{R})$ calculations is normalized such that $\epsilon_{3}^{(3)}=3$ !.

This paper is organized as follows. In section 2 we present our boundary conditions for the spin-3 case in diagonal gauge and derive the asymptotic symmetry algebra as well as higher spin soft hair. In section 3 we map our boundary conditions into highest weight gauge variables and exhibit the twisted Sugawara-like construction. In section 4 we focus on higher spin black holes with soft hair, which we call "higher spin black flowers". We derive their entropy and find that it depends only on our purely gravitational zero modes; in fact, it is linear in them, exactly as in General Relativity. However, if expressed in terms of $W$ algebra currents we recover well-known expressions that involve non-polynomial expressions of spin-2 and spin-3 charges. We further demonstrate that our boundary conditions ensure that all states in our theory are compatible with the regularity conditions. In section 5 we address higher spin black flowers in the metric formalism and achieve full consistency with previous results. In section 6 we generalize some of the main results to spin- $N$, based on $\operatorname{sl}(N, \mathbb{R})$ Chern-Simons theories (with principally embedded $\operatorname{sl}(2, \mathbb{R})$ ). In section 7 we conclude with a discussion and future research directions.

\section{Asymptotic structure}

The asymptotic structure of AdS gravity coupled to higher spin fields in three-dimensional spacetimes was investigated in $[7,8]$, where it was shown that the asymptotic symmetries 
are spanned by two chiral copies of $W$ algebras (see also [9-11]). In order to accommodate the different higher spin black hole solutions in [12, 13], and $[14,15]$, the asymptotic behaviour has to be extended so as to incorporate chemical potentials associated to the global charges. In this sense, alternative proposals have been constructed. The one in $[12$, 16] successfully accommodates the black hole solution with higher spin fields of [12], while the set of boundary conditions in [14, 15] does for the higher spin black holes described therein. It is worth pointing out that the asymptotic symmetries of both sets are different.

Here we construct an inequivalent set of boundary conditions, which reduces to the one recently introduced in [1] when the higher spin fields are switched off. The asymptotic behaviour of the $s l(3, \mathbb{R})$ gauge fields is proposed to be given by

$$
A^{ \pm}=b_{ \pm}^{-1}\left(d+a^{ \pm}\right) b_{ \pm}
$$

so that the dependence on the radial coordinate is completely contained in the group elements

$$
b_{ \pm}=\exp \left( \pm \frac{1}{\ell \zeta^{ \pm}} \mathrm{L}_{1}\right) \cdot \exp \left( \pm \frac{\rho}{2} \mathrm{~L}_{-1}\right) .
$$

The auxiliary connection reads

$$
a^{ \pm}=\left( \pm \mathcal{J}^{ \pm} d \varphi+\zeta^{ \pm} d t\right) \mathrm{L}_{0}+\left( \pm \mathcal{J}_{(3)}^{ \pm} d \varphi+\zeta_{(3)}^{ \pm} d t\right) \mathrm{W}_{0}
$$

where $\mathrm{L}_{i}, \mathrm{~W}_{n}$, with $i=-1,0,1$, and $n=-2,-1,0,1,2$, span the $s l(3, \mathbb{R})$ algebra. Following [14], it can be seen that $\mathcal{J}^{ \pm}$and $\mathcal{J}_{(3)}^{ \pm}$stand for arbitrary functions of (advanced) time and the angular coordinate that correspond to the dynamical fields, while $\zeta^{ \pm}$and $\zeta_{(3)}^{ \pm}$describe their associated Lagrange multipliers that can be assumed to be fixed at the boundary without variation $\left(\delta \zeta^{ \pm}=\delta \zeta_{(3)}^{ \pm}=0\right)$. We shall refer to $\zeta^{ \pm}, \zeta_{(3)}^{ \pm}$as chemical potentials.

The field equations, implying the local flatness of the gauge fields, then reduce to

$$
\dot{\mathcal{J}}^{ \pm}= \pm \zeta^{\prime} \quad \dot{\mathcal{J}}_{(3)}^{ \pm}= \pm \zeta_{(3)}^{\prime}
$$

where dot and prime denote derivatives with respect to $t$ and $\varphi$, respectively.

\subsection{Asymptotic symmetries and the algebra of the canonical generators}

In the canonical approach [17], the variation of the conserved charges

$$
Q\left[\epsilon^{+}, \epsilon^{-}\right]=\mathcal{Q}^{+}\left[\epsilon^{+}\right]-\mathcal{Q}^{-}\left[\epsilon^{-}\right]
$$

associated to gauge symmetries spanned by $\epsilon^{ \pm}=\epsilon_{i}^{ \pm} \mathrm{L}_{i}+\epsilon_{(3) n}^{ \pm} \mathrm{W}_{n}$, that maintain the asymptotic form of the gauge fields, is determined by

$$
\delta \mathcal{Q}^{ \pm}\left[\epsilon^{ \pm}\right]=\mp \frac{k}{4 \pi} \int d \varphi\left(\eta^{ \pm} \delta \mathcal{J}^{ \pm}+\frac{4}{3} \eta_{(3)}^{ \pm} \delta \mathcal{J}_{(3)}^{ \pm}\right)
$$

with $\eta^{ \pm}=\epsilon_{0}^{ \pm}$, and $\eta_{(3)}^{ \pm}=\epsilon_{(3) 0}^{ \pm}$. According to (2.3), the asymptotic symmetries fulfill $\delta_{\epsilon^{ \pm}} a^{ \pm}=d \epsilon^{ \pm}+\left[a^{ \pm}, \epsilon^{ \pm}\right]=\mathcal{O}\left(\delta a^{ \pm}\right)$, provided that the transformation law of the dynamical fields reads

$$
\delta \mathcal{J}^{ \pm}= \pm \eta^{ \pm \prime} \quad \delta \mathcal{J}_{(3)}^{ \pm}= \pm \eta_{(3)}^{ \pm \prime}
$$


and the parameters are time-independent $\left(\dot{\eta}^{ \pm}=\dot{\eta}_{(3)}^{ \pm}=0\right)$. One has to take the quotient over the remaining components of $\epsilon^{ \pm}$, since they just span trivial gauge transformations that neither appear in the variation of the global charges nor in the transformation law of the dynamical fields.

The surface integrals that correspond to the conserved charges associated to the asymptotic symmetries then readily integrate as

$$
\mathcal{Q}^{ \pm}\left[\eta^{ \pm}, \eta_{(3)}^{ \pm}\right]=\mp \frac{k}{4 \pi} \int d \varphi\left(\eta^{ \pm}(\varphi) \mathcal{J}^{ \pm}(\varphi)+\frac{4}{3} \eta_{(3)}^{ \pm}(\varphi) \mathcal{J}_{(3)}^{ \pm}(\varphi)\right)
$$

which are manifestly independent of the radial coordinate $\rho$. Consequently, the boundary could be located at any fixed value $\rho=\rho_{0}$. Hereafter, we assume that $\rho_{0} \rightarrow \infty$, since this choice has the clear advantage of making our analysis to cover the entire spacetime in bulk.

The algebra of the global charges can then be directly obtained from the computation of their Poisson brackets; or as a shortcut, by virtue of $\delta_{Y} Q[X]=\{Q[X], Q[Y]\}$, from the variation of the dynamical fields in (2.7). Expanding in Fourier modes

$$
\mathcal{J}^{ \pm}(\varphi)=\frac{2}{k} \sum_{n=-\infty}^{\infty} J_{n}^{ \pm} e^{ \pm i n \varphi} \quad \mathcal{J}_{(3)}^{ \pm}(\varphi)=\frac{3}{2 k} \sum_{n=-\infty}^{\infty} J_{n}^{(3) \pm} e^{ \pm i n \varphi}
$$

leads to the asymptotic symmetry algebra which is described by a set of $\hat{u}(1)$ currents whose nonvanishing brackets are given by

$$
i\left\{J_{n}^{ \pm}, J_{m}^{ \pm}\right\}=\frac{1}{2} k n \delta_{m+n, 0} \quad i\left\{J_{n}^{(3) \pm}, J_{m}^{(3) \pm}\right\}=\frac{2}{3} k n \delta_{m+n, 0},
$$

with levels $\frac{1}{2} k$, and $\frac{2}{3} k$, respectively.

\section{2 (Higher spin) soft hair}

Following the spin-2 construction [1], we consider now all vacuum descendants $|\psi(q)\rangle$ labelled by a set $q$ of non-negative integers $N^{ \pm}, N_{(3)}^{ \pm}, n_{i}^{ \pm}, n_{i}^{(3) \pm}, m_{i}^{ \pm}$and $m_{i}^{(3) \pm}$

$$
|\psi(q)\rangle=N(q) \prod_{i=1}^{N^{ \pm}}\left(J_{-n_{i}^{ \pm}}^{ \pm}\right)^{m_{i}^{ \pm}} \prod_{i=1}^{N_{(3)}^{ \pm}}\left(J_{-n_{i}^{(3) \pm}}^{(3) \pm}\right)^{m_{i}^{(3) \pm}}|0\rangle .
$$

Here $N(q)$ is some normalization constant such that $\langle\psi(q) \mid \psi(q)\rangle=1$ and the vacuum state ${ }^{1}$ is defined through highest weight conditions, $J_{n}^{ \pm}|0\rangle=J_{n}^{(3) \pm}|0\rangle=0$ for non-negative $n$.

We want to check now if all vacuum descendants $|\psi(q)\rangle$ have the same energy as the vacuum and are thus soft hair (our discussion easily generalizes from soft hair descendants of the vacuum to soft hair descendants of any higher spin black hole state). To this end we consider the surface integral associated with the generator in time, given by

$$
H:=Q\left(\partial_{t}\right)=\frac{k}{4 \pi} \int d \varphi\left(\zeta^{+} \mathcal{J}^{+}+\zeta^{-} \mathcal{J}^{-}+\frac{4}{3} \zeta_{(3)}^{+} \mathcal{J}_{(3)}^{+}+\frac{4}{3} \zeta_{(3)}^{-} \mathcal{J}_{(3)}^{-}\right)
$$

\footnotetext{
${ }^{1}$ The vacuum state corresponds to a massless BTZ black hole. Note that global AdS is not included in the Lorentzian spectrum, since it is gapped by an imaginary amount of the zero mode charges from the vacuum state considered here.
} 
For constant chemical potentials $\zeta^{ \pm}, \zeta_{(3)}^{ \pm}$the field equations (2.4) imply that the dynamical fields become time-independent, and the total Hamiltonian reduces to

$$
H=\zeta^{+} J_{0}^{+}+\zeta^{-} J_{0}^{-}+\zeta_{(3)}^{+} J_{0}^{(3)+}+\zeta_{(3)}^{-} J_{0}^{(3)-},
$$

which clearly commutes with the whole set of asymptotic symmetry generators spanned by $J_{n}^{ \pm}$and $J_{m}^{(3) \pm}$. One then concludes that for an arbitrary fixed value of the total energy, configurations endowed with different sets of nonvanishing $\hat{u}(1)$ charges turn out to be inequivalent, because they can not be related to each other through a pure gauge transformation. Since excitations (2.11) associated with the generators $J_{n}^{ \pm}, J_{m}^{(3) \pm}$ preserve the total energy and cannot be gauged away, they are (higher spin) soft hair in the sense of Hawking, Perry and Strominger [2].

\section{Highest weight gauge and the emergence of composite $W_{3}$ symmetries}

Quite remarkably, it can be seen that spin-2 and spin-3 charges naturally emerge as composite currents constructed out from the $\hat{u}(1)$ ones. Actually, the full set of generators of the $W_{3}$ algebra arises from suitable composite operators of the $\hat{u}(1)$ charges through a twisted Sugawara construction. Here we show this explicitly through the comparison of the new set of boundary conditions proposed in the previous section with the ones that accommodate the higher spin black holes in $[14,15]$, whose asymptotic symmetries are described by two copies of the $W_{3}$ algebra. In order to carry out this task it is necessary to express both sets in terms of the same variables. The asymptotic behaviour described by (2.1) and (2.3) is formulated so that the auxiliary connections $a^{ \pm}$are written in the diagonal gauge, while the set in $[14,15]$ was formulated in the so-called highest weight gauge. Consequently, what we look for can be unveiled once the gauge fields in (2.1) and (2.3) are expressed in terms of the variables that are naturally adapted to the gauge fields $\hat{A}^{ \pm}$in the highest weight gauge.

For a generic choice of Lagrange multipliers, which are still unspecified, the asymptotic form of the gauge fields in the highest weight gauge reads $[14,15]$

$$
\hat{A}^{ \pm}=\hat{b}_{ \pm}^{-1}\left(d+\hat{a}^{ \pm}\right) \hat{b}_{ \pm},
$$

where the radial dependence can be captured by the choice $\hat{b}_{ \pm}=e^{ \pm \rho \mathrm{L}_{0}}$, and

$$
\hat{a}_{\varphi}^{ \pm}=\mathrm{L}_{ \pm 1}-\frac{2 \pi}{k} \mathcal{L}_{ \pm} \mathrm{L}_{\mp 1}-\frac{\pi}{2 k} \mathcal{W}_{ \pm} \mathrm{W}_{\mp 2} \quad \hat{a}_{t}^{ \pm}=\Lambda^{ \pm}\left[\mu_{ \pm}, \nu_{ \pm}\right],
$$

with

$$
\begin{aligned}
\Lambda^{ \pm}\left[\mu_{ \pm}, \nu_{ \pm}\right]= & \pm\left[\mu_{ \pm} \mathrm{L}_{ \pm 1}+\nu_{ \pm} \mathrm{W}_{ \pm 2} \mp \mu_{ \pm}^{\prime} \mathrm{L}_{0} \mp \nu_{ \pm}^{\prime} \mathrm{W}_{ \pm 1}+\frac{1}{2}\left(\mu_{ \pm}^{\prime \prime}-\frac{4 \pi}{k} \mu_{ \pm} \mathcal{L}_{ \pm}+\frac{8 \pi}{k} \mathcal{W}_{ \pm} \nu_{ \pm}\right) \mathrm{L}_{\mp 1}\right. \\
& -\left(\frac{\pi}{2 k} \mathcal{W}_{ \pm} \mu_{ \pm}+\frac{7 \pi}{6 k} \mathcal{L}_{ \pm}^{\prime} \nu_{ \pm}^{\prime}+\frac{\pi}{3 k} \nu_{ \pm} \mathcal{L}_{ \pm}^{\prime \prime}+\frac{4 \pi}{3 \kappa} \mathcal{L}_{ \pm} \nu_{ \pm}^{\prime \prime}-\frac{4 \pi^{2}}{k^{2}} \mathcal{L}_{ \pm}^{2} \nu_{ \pm}-\frac{1}{24} \nu_{ \pm}^{\prime \prime \prime \prime}\right) \mathrm{W}_{\mp 2} \\
& \left.+\frac{1}{2}\left(\nu_{ \pm}^{\prime \prime}-\frac{8 \pi}{k} \mathcal{L}_{ \pm} \nu_{ \pm}\right) \mathrm{W}_{0} \mp \frac{1}{6}\left(\nu_{ \pm}^{\prime \prime \prime}-\frac{8 \pi}{k} \nu_{ \pm} \mathcal{L}_{ \pm}^{\prime}-\frac{20 \pi}{k} \mathcal{L}_{ \pm} \nu_{ \pm}^{\prime}\right) \mathrm{W}_{\mp 1}\right],
\end{aligned}
$$

where $\mathcal{L}_{ \pm}, \mathcal{W}_{ \pm}$and $\mu_{ \pm}, \nu_{ \pm}$stand for arbitrary functions of $t, \varphi$. 
One then needs to find suitable permissible gauge transformations spanned by group elements $g_{ \pm}$, for which $\hat{a}^{ \pm}=g_{ \pm}^{-1}\left(d+a^{ \pm}\right) g_{ \pm}$. These group elements indeed exist, and they are given by $g_{ \pm}=g_{ \pm}^{(1)} g_{ \pm}^{(2)}$, with

$$
\begin{aligned}
& g_{ \pm}^{(1)}=\exp \left[x_{ \pm} \mathrm{L}_{ \pm 1}+y_{ \pm} \mathrm{W}_{ \pm 1}+z_{ \pm} \mathrm{W}_{ \pm 2}\right] \\
& g_{ \pm}^{(2)}=\exp \left[-\frac{1}{2} \mathcal{J}^{ \pm} \mathrm{L}_{\mp 1}-\frac{1}{3} \mathcal{J}_{(3)}^{ \pm} \mathrm{W}_{\mp 1} \pm \frac{1}{6}\left(\mathcal{J}^{ \pm} \mathcal{J}_{(3)}^{ \pm}+\frac{1}{2} \mathcal{J}_{(3)}^{ \pm \prime}\right) \mathrm{W}_{\mp 2}\right]
\end{aligned}
$$

where $x_{ \pm}, y_{ \pm}$and $z_{ \pm}$are arbitrary functions of $t, \varphi$ that fulfill the following conditions:

$$
\begin{aligned}
& x_{ \pm}^{\prime}=1+\mathcal{J}^{ \pm} x_{ \pm}+2 \mathcal{J}_{(3)}^{ \pm} y_{ \pm} \\
& y_{ \pm}^{\prime}=2 \mathcal{J}_{(3)}^{ \pm} x_{ \pm}+\mathcal{J}^{ \pm} y_{ \pm} \\
& z_{ \pm}^{\prime}=2 \mathcal{J}^{ \pm} z_{ \pm} \mp \frac{1}{2} y_{ \pm}
\end{aligned}
$$

with

$$
\begin{aligned}
& \mu_{ \pm}=-x_{ \pm} \zeta^{ \pm}-2 y_{ \pm} \zeta_{(3)}^{ \pm} \pm \dot{x}_{ \pm} \pm \frac{4}{3} \nu_{ \pm} \mathcal{J}_{(3)}^{ \pm} \\
& \nu_{ \pm}=-2 \zeta_{ \pm} z_{ \pm} \pm\left(x_{ \pm}^{2}-y_{ \pm}^{2}\right) \zeta_{(3)}^{ \pm}+\frac{1}{2}\left(y_{ \pm} \dot{x}_{ \pm}-x_{ \pm} \dot{y}_{ \pm} \pm 2 \dot{z}_{ \pm}\right)
\end{aligned}
$$

Consistency of eqs. (3.5) and (3.6) on-shell implies that the Lagrange multipliers in the highest weight gauge, $\mu_{ \pm}, \nu_{ \pm}$, depend not only on the Lagrange multipliers in the diagonal gauge, $\zeta^{ \pm}, \zeta_{(3)}^{ \pm}$, but also on their corresponding global charges $\mathcal{J}^{ \pm}, \mathcal{J}_{(3)}^{ \pm}$, according to

$$
\begin{aligned}
& \pm \frac{2}{3} \mathcal{J}_{(3)}^{ \pm} \nu_{ \pm}^{\prime} \mp \frac{8}{3}\left(\mathcal{J}^{ \pm} \mathcal{J}_{(3)}^{ \pm}+\frac{1}{2} \mathcal{J}_{(3)}^{ \pm \prime}\right) \nu_{ \pm}+\mu_{ \pm}^{\prime}-\mu_{ \pm} \mathcal{J}^{ \pm}=-\zeta^{ \pm} \\
& \frac{1}{2} \nu_{ \pm}^{\prime \prime}-\frac{3}{2} \mathcal{J}_{ \pm} \nu_{ \pm}^{\prime}+\left(\left(\mathcal{J}^{ \pm}\right)^{2}-\frac{4}{3}\left(\mathcal{J}_{(3)}^{ \pm}\right)^{2}-\mathcal{J}^{ \pm \prime}\right) \nu_{ \pm} \pm \mu_{ \pm} \mathcal{J}_{(3)}^{ \pm}= \pm \zeta_{(3)}^{ \pm}
\end{aligned}
$$

The gauge fields $a^{ \pm}$and $\hat{a}^{ \pm}$are then mapped to each other provided

$$
\begin{aligned}
\mathcal{L}_{ \pm} & = \pm \frac{k}{4 \pi}\left(\frac{1}{2}\left(\mathcal{J}^{ \pm}\right)^{2}+\frac{2}{3}\left(\mathcal{J}_{(3)}^{ \pm}\right)^{2}+\mathcal{J}^{ \pm \prime}\right) \\
\mathcal{W}_{ \pm} & =\mp \frac{k}{6 \pi}\left(-\frac{8}{9}\left(\mathcal{J}_{(3)}^{ \pm}\right)^{3}+2\left(\mathcal{J}^{ \pm}\right)^{2} \mathcal{J}_{(3)}^{ \pm}+\mathcal{J}_{(3)}^{ \pm} \mathcal{J}^{ \pm \prime}+3 \mathcal{J}^{ \pm} \mathcal{J}_{(3)}^{ \pm \prime}+\mathcal{J}_{(3)}^{ \pm \prime \prime}\right)
\end{aligned}
$$

from which one recognizes the Miura transformation between the variables, see e.g. [18].

In sum, our proposal for boundary conditions once expressed in the highest weight gauge, is such that the Lagrange multipliers $\mu_{ \pm}$and $\nu_{ \pm}$depend on the dynamical variables according to (3.7) and (3.8), where $\zeta^{ \pm}$and $\zeta_{(3)}^{ \pm}$are assumed to be fixed without variation $\left(\delta \zeta^{ \pm}=\delta \zeta_{(3)}^{ \pm}=0\right)$. Note that the functions $\mathcal{L}_{ \pm}, \mathcal{W}_{ \pm}$, that are naturally defined in the highest weight gauge, depend on the global charges $\mathcal{J}^{ \pm}, \mathcal{J}_{(3)}^{ \pm}$as in eqs. (3.9), (3.10). 
Indeed, for a generic choice of Lagrange multipliers in the highest weight gauge, the field equations read [15]

$$
\begin{aligned}
\dot{\mathcal{L}}_{ \pm}= & \pm 2 \mathcal{L}_{ \pm} \mu_{ \pm}^{\prime} \pm \mu_{ \pm} \mathcal{L}_{ \pm}^{\prime} \mp \frac{k}{4 \pi} \mu_{ \pm}^{\prime \prime \prime} \mp 2 \nu_{ \pm} \mathcal{W}_{ \pm}^{\prime} \mp 3 \mathcal{W}_{ \pm} \nu_{ \pm}^{\prime} \\
\dot{\mathcal{W}}_{ \pm}= & \pm 3 \mathcal{W}_{ \pm} \mu_{ \pm}^{\prime} \pm \mu_{ \pm} \mathcal{W}_{ \pm}^{\prime} \pm \frac{2}{3} \nu_{ \pm}\left(\mathcal{L}_{ \pm}^{\prime \prime \prime}-\frac{16 \pi}{k} \mathcal{L}_{ \pm}^{2 \prime}\right) \pm 3\left(\mathcal{L}_{ \pm}^{\prime \prime}-\frac{64 \pi}{9 k} \mathcal{L}_{ \pm}^{2}\right) \nu_{ \pm}^{\prime} \\
& \pm 5 \nu_{ \pm}^{\prime \prime} \mathcal{L}_{ \pm}^{\prime} \pm \frac{10}{3} \mathcal{L}_{ \pm} \nu_{ \pm}^{\prime \prime \prime} \mp \frac{k}{12 \pi} \nu_{ \pm}^{(5)}
\end{aligned}
$$

which by virtue of the definition of our boundary conditions, in eqs. (3.7), (3.8) and (3.9), (3.10), reduce to the remarkably simple ones, given by $\dot{\mathcal{J}}^{ \pm}= \pm \zeta^{\prime}, \dot{\mathcal{J}}_{(3)}^{ \pm}= \pm \zeta_{(3)}^{\prime}$, which were directly obtained in the diagonal gauge (see eq. (2.4)).

It is also worth highlighting that eqs. (3.9), (3.10) can be regarded as the higher spin gravity version of the twisted Sugawara construction. In fact, we show now that the currents $\mathcal{L}_{ \pm}, \mathcal{W}_{ \pm}$fulfill the $W_{3}$ algebra. Let us recall that according to (2.7), the transformation law of the dynamical fields under the $\hat{u}(1)$ asymptotic symmetries reads $\delta \mathcal{J}^{ \pm}= \pm \eta^{ \pm \prime}$, $\delta \mathcal{J}_{(3)}^{ \pm}= \pm \eta_{(3)}^{ \pm \prime}$. Besides, the relationship for the Lagrange multipliers in (3.7), (3.8) implies that the corresponding one for the parameters in the highest weight gauge, defined as $\varepsilon_{ \pm}, \chi_{ \pm}$, with the Lagrange multipliers and their associated charges as formulated in the diagonal gauge, given by $\eta^{ \pm}, \eta_{(3)}^{ \pm}$, and $\mathcal{J}^{ \pm}, \mathcal{J}_{(3)}^{ \pm}$, respectively, reads

$$
\begin{aligned}
& \pm \frac{2}{3} \mathcal{J}_{(3)}^{ \pm} \chi_{ \pm}^{\prime} \mp \frac{8}{3}\left(\mathcal{J}^{ \pm} \mathcal{J}_{(3)}^{ \pm}+\frac{1}{2} \mathcal{J}_{(3)}^{ \pm \prime}\right) \chi_{ \pm}+\varepsilon_{ \pm}^{\prime}-\varepsilon_{ \pm} \mathcal{J}^{ \pm}=-\eta^{ \pm} \\
& \frac{1}{2} \chi_{ \pm}^{\prime \prime}-\frac{3}{2} \mathcal{J}_{ \pm} \chi_{ \pm}^{\prime}+\left(\left(\mathcal{J}^{ \pm}\right)^{2}-\frac{4}{3}\left(\mathcal{J}_{(3)}^{ \pm}\right)^{2}-\mathcal{J}^{ \pm \prime}\right) \chi_{ \pm} \pm \varepsilon_{ \pm} \mathcal{J}_{(3)}^{ \pm}= \pm \eta_{(3)}^{ \pm} .
\end{aligned}
$$

Therefore, the transformation laws for $\mathcal{L}_{ \pm}$and $\mathcal{W}_{ \pm}$can be directly read from (3.9), (3.10), which reduce to

$$
\begin{aligned}
\delta \mathcal{L}_{ \pm}= & \pm 2 \mathcal{L}_{ \pm} \varepsilon_{ \pm}^{\prime} \pm \varepsilon_{ \pm} \mathcal{L}_{ \pm}^{\prime} \mp \frac{k}{4 \pi} \varepsilon_{ \pm}^{\prime \prime \prime} \mp 2 \chi_{ \pm} \mathcal{W}_{ \pm}^{\prime} \mp 3 \mathcal{W}_{ \pm} \chi_{ \pm}^{\prime} \\
\delta \mathcal{W}_{ \pm}= & \pm 3 \mathcal{W}_{ \pm} \varepsilon_{ \pm}^{\prime} \pm \varepsilon_{ \pm} \mathcal{W}_{ \pm}^{\prime} \pm \frac{2}{3} \chi_{ \pm}\left(\mathcal{L}_{ \pm}^{\prime \prime \prime}-\frac{16 \pi}{k} \mathcal{L}_{ \pm}^{2 \prime}\right) \pm 3\left(\mathcal{L}_{ \pm}^{\prime \prime}-\frac{64 \pi}{9 k} \mathcal{L}_{ \pm}^{2}\right) \chi_{ \pm}^{\prime} \\
& \pm 5 \chi_{ \pm}^{\prime \prime} \mathcal{L}_{ \pm}^{\prime} \pm \frac{10}{3} \mathcal{L}_{ \pm} \chi_{ \pm}^{\prime \prime \prime} \mp \frac{k}{12 \pi} \chi_{ \pm}^{(5)}
\end{aligned}
$$

It is then apparent that $\mathcal{L}_{ \pm}$and $\mathcal{W}_{ \pm}$turn out to be composite anomalous spin-2 and spin3 currents, respectively. In other words, the asymptotic $W_{3}$ algebra obtained in $[14,15]$ for a different set of boundary conditions, being defined through requiring the Lagrange multipliers in the highest weight gauge to be fixed without variation $\left(\delta \mu_{ \pm}=\delta \nu_{ \pm}=0\right)$, is recovered as a composite one that emerges from the $\hat{u}(1)$ currents.

Despite of the fact that the spin-2 and spin-3 currents $\mathcal{L}_{ \pm}, \mathcal{W}_{ \pm}$fulfill the $W_{3}$ algebra, their associated global charges generate the $\hat{u}(1)$ current algebras discussed in section 2 . This is so because, by virtue of eqs. in (3.13) and (3.9), (3.10) the variation of the global 
charges readily reduces to

$$
\delta \mathcal{Q}^{ \pm}=\mp \int d \varphi\left(\varepsilon_{ \pm} \delta \mathcal{L}_{ \pm}-\chi_{ \pm} \delta \mathcal{W}_{ \pm}\right)=\mp \frac{k}{4 \pi} \int d \varphi\left(\eta^{ \pm} \delta \mathcal{J}^{ \pm}+\frac{4}{3} \eta_{(3)}^{ \pm} \delta \mathcal{J}_{(3)}^{ \pm}\right)
$$

so that they satisfy the current algebras in (2.10). Indeed, this result just reflects the fact that the gauge transformation that maps our asymptotic conditions in the highest weight and diagonal gauges, spanned by the group element defined through (3.4), is a permissible one in the sense of [15]. Therefore, the global charges associated to our asymptotic conditions, although written in the highest weight gauge as in eqs. (3.7), (3.8) and (3.9), (3.10), manifestly do not fulfill the $W_{3}$ algebra. This is because the Lagrange multipliers $\mu_{ \pm}, \nu_{ \pm}$, are not chosen to be fixed at infinity without variation as in [14, 15], but instead, here they explicitly depend on the global charges. What is actually kept fixed at the boundary without variation is the set of Lagrange multipliers that is naturally defined in the diagonal gauge $\left(\delta \zeta^{ \pm}=\delta \zeta_{(3)}^{ \pm}=0\right)$.

\section{Higher spin black holes with soft hair}

In this section we discuss higher spin black hole solutions with soft hair, address their regularity and calculate their entropy. In section 4.1 we present our results in diagonal gauge, which is most suitable for our boundary conditions. In section 4.2 we discuss our results in highest weight gauge that is used traditionally in higher spin theories.

\subsection{Regularity and black hole entropy in diagonal gauge}

As shown in section 2.2, the simpler subset of our boundary conditions, obtained by choosing the Lagrange multipliers $\zeta^{ \pm}, \zeta_{(3)}^{ \pm}$to be constants, possesses the noticeable property of making the global charges $J_{n}^{ \pm}, J_{m}^{(3) \pm}$ to behave as (higher spin) soft hair. An additional remarkable feature that also occurs in this case is the fact that regularity of the whole spectrum of Euclidean solutions that fulfill our boundary conditions holds everywhere, regardless the value of the global charges.

The entire space of solutions of the field equations (2.4) that satisfies our boundary conditions in this case is given by $\mathcal{J}^{ \pm}=\mathcal{J}^{ \pm}(\varphi), \mathcal{J}_{(3)}^{ \pm}=\mathcal{J}_{(3)}^{ \pm}(\varphi)$, which generically describes stationary non spherically symmetric higher spin black flowers endowed with all the possible left and right $\hat{u}(1)$ charges. In order to see this explicitly, we assume that the topology of the Euclidean manifold is the one of a solid torus, where the Euclidean time coordinate $\tau=i t$ corresponds to the contractible cycle. As explained in $[14,15]$, the Lagrange multipliers correspond to the chemical potentials associated to the global charges (see below), and since in our case they are all switched on, the range of the coordinates can be fixed once and for all. Here we assume that the boundary of the solid torus is described by a trivial modular parameter, so that $0 \leq \varphi<2 \pi, 0 \leq \tau<\beta$, where $\beta=T^{-1}$ is the inverse of the Hawking temperature.

Regularity of the Euclidean solution then requires the holonomy of the gauge fields along any contractible cycle $\mathcal{C}$ to be trivial, which reads

$$
\mathcal{H}_{\mathcal{C}}=\mathcal{P} e^{\int_{\mathcal{C}} a}=\mathbb{1} .
$$


Since the general solution is naturally formulated in the diagonal gauge, as in (2.3), the regularity condition (4.1) is trivially solved by

$$
\zeta^{ \pm}=\frac{2 \pi n}{\beta}+\frac{2}{3} \zeta_{(3)}^{ \pm} \quad \zeta_{(3)}^{ \pm}=\frac{3}{2} \frac{\pi}{\beta} m
$$

with $m, n$ being integers. Hence, regularity holds regardless the value of the global charges.

The generic regular solution then carries the entire set of $\hat{u}(1)$ charges spanned by $J_{n}^{ \pm}, J_{m}^{(3) \pm}$. Therefore, by virtue of the map between the $\hat{u}(1)$ and $W_{3}$ currents, defined through (3.9), (3.10), this class of solutions is endowed with nontrivial spin-2 and spin-3 (composite) charges.

This class of static non-spherically symmetric solutions are higher spin black flowers, whose ripples cannot be gauged away because they are characterized by their corresponding (higher spin) soft hair charges.

The simplest case in which the solution is only endowed with the zero-modes charges $J_{0}^{ \pm}, J_{0}^{(3) \pm}$, reduces to the stationary spherically symmetric higher spin black hole solution discussed in $[14,15]$.

Generic higher spin black holes with soft hair can be obtained from the spherically symmetric one aforementioned by acting on it with an arbitrary soft boost, which corresponds to applying a generic element of the asymptotic symmetry group globally. Thus, as explained in section 2.2, the action of the $\hat{u}(1)$ generators does not change the total energy, and noteworthy, the generic solution obtained through this procedure remains regular. This is a peculiar feature of our boundary conditions, which does not hold for different choices of Lagrange multipliers. Indeed, for the choice in $[14,15]$, in which the Lagrange multipliers in the highest weight gauge are chosen to be constant, if one applies the same procedure starting from a spherically symmetric solution, one finds that under the action of the $W_{3}$ symmetries not only the energy changes, but the regularity of the $W_{3}$-boosted solution is generically spoiled. This reflects the fact that the additional $W_{3}$ charges of the boosted solution do not correspond to soft hair, since they do not commute with the Hamiltonian.

As shown in [19, 20], the correct expression for the black hole entropy in the context of higher spin gravity can be obtained from

$$
\delta S=\beta \delta H=-\frac{k_{N}}{\pi} \operatorname{Im}\left(\beta \int d \varphi \operatorname{tr}\left[a_{\tau} \delta a_{\varphi}\right]\right) .
$$

Under certain reasonable assumptions, which are fulfilled by our set of boundary conditions expressed in the diagonal gauge, according to [15, 21], the variation of the entropy integrates as

$$
S=-\frac{k_{N}}{\pi} \operatorname{Im}\left(\beta \int d \varphi \operatorname{tr}\left[a_{\tau} a_{\varphi}\right]\right) .
$$

The entropy of a generic higher spin black flower can then be readily obtained from (4.4), which by virtue of (2.3) and (4.2), in the Lorentzian case reads

$$
S=\pi\left[(2 n+m)\left(J_{0}^{+}+J_{0}^{-}\right)+3 m\left(J_{0}^{(3)+}+J_{0}^{(3)-}\right)\right] .
$$


Note that the entropy in (4.5) only captures the electric-like zero-mode charges, and hence it does not depend on (higher spin) soft hair.

An interesting effect occurs for the branch of higher spin black flowers that is continuously connected to the BTZ black hole [22,23], corresponding to $m=0, n=1$. Indeed, for this branch the entropy (4.5) is found to depend just on the zero modes of the electric-like $\hat{u}(1)$ charges of the purely gravitational sector, i.e.,

$$
S=2 \pi\left(J_{0}^{+}+J_{0}^{-}\right)
$$

Nonetheless, the information about the presence of the higher spin fields is subtlety hidden within the purely gravitational global charges, as it can be seen from the map between the $\hat{u}(1)$ and $W_{3}$ currents. In fact, for the spherically symmetric higher spin black hole, by virtue of (3.9), (3.10), the relationship between the zero modes of the purely gravitational $\hat{u}(1)$ charges and the zero modes of the $W_{3}$ ones reads

$$
J_{0}^{ \pm}=\sqrt{2 \pi k \mathcal{L}_{ \pm}} \cos \left[\frac{1}{3} \arcsin \left(\frac{3}{8} \sqrt{\frac{3 k}{2 \pi \mathcal{L}_{ \pm}^{3}}} \mathcal{W}_{ \pm}\right)\right] .
$$

Therefore, replacing (4.7) into (4.6) one recovers the following expression for the higher spin black hole entropy in terms of the spin-2 and spin-3 charges, which reads

$$
\begin{aligned}
S= & 2 \pi \sqrt{2 \pi k}\left(\sqrt{\mathcal{L}_{+}} \cos \left[\frac{1}{3} \arcsin \left(\frac{3}{8} \sqrt{\frac{3 k}{2 \pi \mathcal{L}_{+}^{3}}} \mathcal{W}_{+}\right)\right]\right. \\
& \left.+\sqrt{\mathcal{L}_{-}} \cos \left[\frac{1}{3} \arcsin \left(\frac{3}{8} \sqrt{\frac{3 k}{2 \pi \mathcal{L}_{-}^{3}}} \mathcal{W}_{-}\right)\right]\right),
\end{aligned}
$$

in full agreement with the result obtained in [15].

\subsection{Remarks on regularity and entropy in highest weight gauge}

As explained in section 3, our boundary conditions can be expressed in terms of the natural variables of the highest weight gauge by choosing the Lagrange multipliers $\mu_{ \pm}, \nu_{ \pm}$according to (3.7), (3.8), as well as mapping the $W_{3}$ currents $\mathcal{L}_{ \pm}, \mathcal{W}_{ \pm}$in terms of the $\hat{u}(1)$ ones as in (3.9), (3.10). Despite that the formulation of our boundary conditions in the highest weight gauge is certainly more involved than in the diagonal one, it is worth mentioning that the computations related to regularity of the Euclidean higher spin black flowers and their entropy can be carried out anyway, by taking into account certain refinements. Indeed, in the highest weight gauge, the left hand side of the regularity condition (4.1) cannot be so easily exponentiated. Nevertheless, the regularity condition can be alternatively written as

$$
\begin{aligned}
& \beta^{2} \operatorname{tr}\left[\hat{a}_{\tau}^{2}\right]=-8 \pi^{2}\left(m^{2}+m n+n^{2}\right) \\
& \beta^{3} \operatorname{det}\left[\hat{a}_{\tau}\right]=8 \pi^{3} i(m+n) m n,
\end{aligned}
$$

where $\hat{a}_{\tau}$ stands for the Euclidean continuation of $\hat{a}_{t}^{ \pm}$in $(3.2)$, with $\Lambda^{ \pm}\left[\mu_{ \pm}, \nu_{ \pm}\right]$given by (3.3). The latter equations actually become extremely convoluted, since $\operatorname{tr}\left[\hat{a}_{\tau}^{2}\right]$ and 
$\operatorname{det}\left[\hat{a}_{\tau}\right]$ evaluate as

$$
\begin{aligned}
\operatorname{tr}\left[\hat{a}_{\tau}^{2}\right]= & \frac{48 \pi}{k}\left(\nu \mathcal{W}-\frac{1}{3} \mu \mathcal{L}+\frac{k}{12 \pi} \mu^{\prime \prime}\right) \mu-\frac{2^{9} \pi^{2}}{3 k^{2}}\left[\left(\mathcal{L}^{2}-\frac{k}{16 \pi} \mathcal{L}^{\prime \prime}\right) \nu\right. \\
& \left.-\frac{5 k}{2^{5} \pi}\left(\nu^{\prime} \mathcal{L}^{\prime}+2 \nu^{\prime \prime} \mathcal{L}-\frac{k}{20 \pi} \nu^{\prime \prime \prime \prime}\right)\right] \nu-\frac{2}{3} \nu^{\prime \prime 2}-2 \mu^{\prime 2}+\frac{4}{3}\left(\nu^{\prime \prime \prime}-\frac{20 \pi}{k} \nu^{\prime} \mathcal{L}\right) \nu^{\prime} \\
i \operatorname{det}\left[\hat{a}_{\tau}\right]= & \frac{2^{7} \pi^{2}}{3 k^{2}}\left[\nu^{2} \mathcal{L}-\frac{3 k}{32 \pi}\left(\mu^{2}-\nu^{\prime 2}+\frac{4}{3} \nu \nu^{\prime \prime}\right)\right]\left[\mu \mathcal{W}+\frac{2}{3} \nu\left(\mathcal{L}^{\prime \prime}-\frac{12 \pi}{k} \mathcal{L}^{2}\right)\right. \\
& \left.+\frac{7}{3} \nu^{\prime} \mathcal{L}^{\prime}+\frac{8}{3} \nu^{\prime \prime} \mathcal{L}-\frac{k}{12 \pi} \nu^{\prime \prime \prime \prime}\right]+\frac{2^{5} \pi^{2}}{3 k^{2}}\left[\left(\mu-\frac{5}{3} \nu^{\prime}\right) \mathcal{L}-2\left(\mathcal{W}+\frac{1}{3} \mathcal{L}^{\prime}\right) \nu\right. \\
& \left.-\frac{k}{4 \pi}\left(\mu-\frac{1}{3} \nu^{\prime}\right)^{\prime \prime}\right]\left[\left(\mu-\nu^{\prime}\right)\left(\nu \mathcal{L}-\frac{3 k}{8 \pi}\left(\mu+\frac{1}{3} \nu^{\prime}\right)^{\prime}\right)\right. \\
& \left.-6 \nu\left(\left(\mathcal{W}-\frac{1}{3} \mathcal{L}^{\prime}\right) \nu-\frac{1}{2}\left(\mu+\frac{5}{3} \nu^{\prime}\right) \mathcal{L}+\frac{k}{8 \pi}\left(\mu+\frac{1}{3} \nu^{\prime}\right)^{\prime \prime}\right)\right] \\
& +\frac{2^{3} \pi}{k}\left(\mu^{\prime}-\frac{1}{3}\left(\nu^{\prime \prime}-\frac{8 \pi}{k} \nu \mathcal{L}\right)\right)\left[( \mu + \nu ^ { \prime } ) \left(\nu\left(\mathcal{W}-\frac{1}{3} \mathcal{L}^{\prime}\right)-\frac{1}{2}\left(\mu+\frac{5}{3} \nu^{\prime}\right) \mathcal{L}\right.\right. \\
& \left.\left.+\frac{k}{8 \pi}\left(\mu+\frac{1}{3} \nu^{\prime}\right)^{\prime \prime}\right)-\frac{2^{4} \pi}{3^{2} k}\left(\nu \mathcal{L}-\frac{k}{8 \pi} \nu^{\prime \prime}\right)\left(\nu \mathcal{L}-\frac{3 k}{8 \pi}\left(\mu+\frac{1}{3} \nu^{\prime}\right)\right)\right]
\end{aligned}
$$

respectively. Nonetheless, after some amount of algebraic work, one verifies that the regularity conditions in (4.9) conspire with the expressions that define our boundary conditions in the highest weight gauge, given by (3.7), (3.8), and (3.9), (3.10), so that regularity holds provided the equations in (4.2) do, in full agreement with the result that was easily performed in the diagonal gauge.

The formulation of our boundary conditions in the highest weight gauge is such that the hypotheses assumed in $[15,21]$ do not apply, and hence the entropy formula in (4.4) cannot be used in this case, since it would yield an incorrect result. However, the higher spin black flower entropy can still be obtained through the original formula in $(4.3)[19,20]$. Indeed, replacing $\hat{a}_{\varphi}^{ \pm}$and $\hat{a}_{t}^{ \pm}$into the variation of the (Lorentzian) entropy, one obtains

$$
\begin{aligned}
\delta S & =\frac{k_{3}}{2 \pi} \beta \int d \varphi\left(\operatorname{tr}\left[\hat{a}_{\tau}^{+} \delta \hat{a}_{\varphi}^{+}\right]-\operatorname{tr}\left[\hat{a}_{\tau}^{-} \delta \hat{a}_{\varphi}^{-}\right]\right) \\
& =\int d \varphi\left(\mu_{+} \delta \mathcal{L}_{+}+\mu_{-} \delta \mathcal{L}_{-}-\nu_{+} \delta \mathcal{W}_{+}-\nu_{-} \delta \mathcal{W}_{-}\right)
\end{aligned}
$$

which by virtue of (3.7), (3.8), and (3.9), (3.10), reduces to

$$
\delta S=\frac{k_{3}}{4 \pi} \beta \int d \varphi\left(\zeta^{+} \delta \mathcal{J}^{+}+\zeta^{-} \delta \mathcal{J}^{-}+\frac{4}{3} \zeta_{(3)}^{+} \delta \mathcal{J}_{(3)}^{+}+\frac{4}{3} \zeta_{(3)}^{-} \delta \mathcal{J}_{(3)}^{-}\right)
$$

Since, $\zeta^{ \pm}$and $\zeta_{(3)}^{ \pm}$are assumed to be constants, the variation of the entropy readily integrates as

$$
S=\frac{k_{3}}{4 \pi} \beta \int d \varphi\left(\zeta^{+} \mathcal{J}^{+}+\zeta^{-} \mathcal{J}^{-}+\frac{4}{3} \zeta_{(3)}^{+} \mathcal{J}_{(3)}^{+}+\frac{4}{3} \zeta_{(3)}^{-} \mathcal{J}_{(3)}^{-}\right)
$$

so that once the regularity conditions in (4.2) are taken into account, the entropy reduces to the formula in eq. (4.5) which was straightforwardly obtained in the diagonal gauge. 


\section{Higher spin black holes with soft hair in the metric formalism}

The entropy of the higher spin black flower for the branch that is connected with the BTZ black hole in eq. (4.6) can also be alternatively recovered in the metric formalism. In order to deal with non spherically symmetric horizons, the formula proposed in [20] has to be slightly refined, so that the entropy reads

$$
S=\frac{1}{4 G} \int_{\partial \Sigma_{+}} \mathcal{A} \cos \left[\frac{1}{3} \arcsin \left(\left(\sqrt{3} \frac{\phi}{\mathcal{A}}\right)^{3}\right)\right] d \sigma,
$$

where $\mathcal{A}$ and $\phi$ stand for the horizon area element and its spin-3 analogue, respectively. They are naturally defined in terms of the pullback of the metric and the spin-3 field at the spacelike section of the horizon, so that their integrals become reparametrization invariant, i.e.,

$$
\begin{aligned}
& A=\int_{\partial \Sigma_{+}} \mathcal{A} d \sigma=\int_{\partial \Sigma_{+}}\left(g_{\mu \nu} \frac{d x^{\mu}}{d \sigma} \frac{d x^{\nu}}{d \sigma}\right)^{1 / 2} d \sigma \\
& \Phi=\int_{\partial \Sigma_{+}} \phi d \sigma=\int_{\partial \Sigma_{+}}\left(\Phi_{\mu \nu \rho} \frac{d x^{\mu}}{d \sigma} \frac{d x^{\nu}}{d \sigma} \frac{d x^{\rho}}{d \sigma}\right)^{1 / 3} d \sigma .
\end{aligned}
$$

It is worth mentioning that, although a full nonperturbative action principle for higher spin gravity formulated exclusively in term of the metric and the spin-3 field is still unknown, the entropy in (5.1) can be written as a closed analytic formula. In the weak spin-3 limit, an explicit action principle was constructed in [24] up to quadratic order, from which the entropy in the static case was also obtained and it turns out to agree with the corresponding perturbative expansion of (5.1).

The higher spin black flower metric corresponds to a generalization of the soft hairy black holes recently obtained in [1] for General Relativity with negative cosmological constant in vacuum. In order to explicitly see the contact, for simplicity, let us choose the Lagrange multipliers to be constants according to $\zeta^{ \pm}=-a, \zeta_{(3)}^{ \pm}=-a_{(3)}$. The spacetime metric can then be reconstructed from the gauge fields as in (1.4), and it is found to be given by

$$
\begin{aligned}
d s^{2}= & -2 \rho \ell f(\rho) a\left(1+4(1-2 f(\rho))^{2} a^{-2} a_{(3)}^{2}\right) d v^{2}+2 \ell d \rho d v-2 \omega a^{-1} d \rho d \varphi \\
& +4\left(\omega+4(1-2 f(\rho))^{2} \omega_{(3)} a^{-1} a_{(3)}\right) \rho f(\rho) d v d \varphi \\
& +\left[\gamma^{2}+\frac{4}{3} \gamma_{(3)}^{2}+\frac{2 \rho}{\ell} a^{-1} f(\rho)\left(\gamma^{2}-\omega^{2}+4(1-2 f(\rho))^{2}\left(\gamma_{(3)}^{2}-\omega_{(3)}^{2}\right)\right)\right] d \varphi^{2},
\end{aligned}
$$

with $t=v$, and $f(\rho)=1+\frac{\rho}{2 a \ell}$. The remaining arbitrary functions are related to the global charges according to

$$
\ell \mathcal{J}^{ \pm}:=\gamma \pm \omega ; \quad \ell \mathcal{J}_{(3)}^{ \pm}:=\gamma_{(3)} \pm \omega_{(3)},
$$

which means that they cannot be gauged away by proper gauge transformations. The event horizon is located at $\rho=0$. The line-element (5.4) shows that our boundary conditions 
can be interpreted as near horizon conditions that for small $\rho$ recover Rindler space in Eddington-Finkelstein type of coordinates. In the case of $\omega_{(3)}=a_{(3)}=0$, the spacetime geometry reduces to the one found in [1] which is of negative constant curvature.

Analogously, the spin-3 field can be reconstructed from (1.4), whose explicit form is given in the appendix B. For our purposes, it is enough to know the behaviour of its purely angular component around the horizon, which reads

$$
\Phi_{\varphi \varphi \varphi}=-\frac{2}{27}\left(4 \gamma_{(3)}^{2}-9 \gamma^{2}\right) \gamma_{(3)}+\mathcal{O}(\rho) .
$$

Note that in three spacetime dimensions the metric component $g_{\rho \varphi}$ can always be gauged away. Indeed, even in the case of a generic choice of Lagrange multipliers $\zeta^{ \pm}$, $\zeta_{(3)}^{ \pm}$, the metric and the spin-3 field can also be directly reconstructed from (1.4) in normal coordinates. This can be done through a permissible gauge transformation, which amounts to replace the group elements $b_{ \pm}$in $(2.2)$ by $b_{ \pm}=e^{ \pm \frac{r}{2}\left(\mathrm{~L}_{1}+\mathrm{L}_{-1}\right)}$. The line element then reads

$$
\begin{aligned}
d s^{2}= & -\frac{\ell^{2}}{2}\left[\zeta_{(3)}^{+} \zeta_{(3)}^{-}\left(\cosh (4 r)+\frac{5}{3}\right)+2 \zeta^{+} \zeta^{-} \cosh ^{2}(r)-\frac{1}{2}\left(\zeta^{+}+\zeta^{-}\right)^{2}\right. \\
& \left.-\frac{2}{3}\left(\zeta_{(3)}^{+}+\zeta_{(3)}^{-}\right)^{2}\right] d t^{2}+\ell^{2} d r^{2}+\ell\left[\frac{1}{2}\left(\zeta_{(3)}^{+}-\zeta_{(3)}^{-}\right) \gamma_{(3)}\left(\cosh (4 r)+\frac{5}{3}\right)\right. \\
& \left.-\left(\zeta_{(3)}^{+}+\zeta_{(3)}^{-}\right) \omega_{(3)} \sinh ^{2}(2 r)-\left(\zeta^{+}+\zeta^{-}\right) \omega \sinh ^{2}(r)+\left(\zeta^{+}-\zeta^{-}\right) \gamma \cosh ^{2}(r)\right] d t d \varphi \\
& +\left[\frac{1}{2}\left(\cosh (4 r)+\frac{5}{3}\right) \gamma_{(3)}^{2}-\omega_{(3)}^{2} \sinh ^{2}(2 r)+\gamma^{2} \cosh ^{2}(r)-\omega^{2} \sinh ^{2}(r)\right] d \varphi^{2},
\end{aligned}
$$

so that the event horizon locates at $r=0$. This class of geometries asymptotically approaches to locally $\mathrm{AdS}_{3}$ spacetimes of radius $\ell / 2$.

The explicit form of the spin-3 field is written in the appendix B, and its purely angular component close to the horizon behaves as equation (5.6) with $\mathcal{O}(\rho)$ replaced by $\mathcal{O}\left(r^{2}\right)$. It is then simple to verify that requiring regularity of the Euclidean metric and the spin-3 field around the horizon fixes the Lagrange multipliers (chemical potentials) precisely as in eq. (4.2) with $m=0$ and $n=1$, i.e., for the branch that is connected to the BTZ black hole. Further issues about regularity of the fields in the metric formalism have been discussed in [12, 25].

For the most generic higher spin black flower configuration (5.7), and (the again transformed) (5.6), as well as for the particular case in (5.4), (5.6), the event horizon area element and its spin-3 analogue in eqs. (5.2), (5.3), respectively, then evaluate as

$$
\begin{aligned}
\mathcal{A}^{2} & =\left.g_{\varphi \varphi}\right|_{r_{+}}=\gamma^{2}+\frac{4}{3} \gamma_{(3)}^{2} \\
\phi^{3} & =\left.\Phi_{\varphi \varphi \varphi}\right|_{r_{+}}=\frac{2}{27} \gamma_{(3)}\left(9 \gamma^{2}-4 \gamma_{(3)}^{2}\right),
\end{aligned}
$$

so that, by virtue of the identity

$$
\cos \left[\frac{1}{3} \arcsin \left(\frac{x\left(x^{2}-3\right)}{\left(x^{2}+1\right)^{3 / 2}}\right)\right]=\frac{1}{\sqrt{x^{2}+1}}
$$


the entropy in (5.1) reduces to

$$
S=\frac{1}{4 G} \int \gamma d \varphi=\frac{k}{2} \int\left(\mathcal{J}^{+}+\mathcal{J}^{-}\right) d \varphi=2 \pi\left(J_{0}^{+}+J_{0}^{-}\right)
$$

in full agreement with the result found exclusively in terms of gauge fields in section 4 (see eq. (4.6)).

Note that $\int \gamma d \varphi$ could then be regarded as a sort of "effective higher spin horizon area", which curiously corresponds to the horizon area of the purely gravitational black flower in the absence of higher spin fields.

\section{Extension to $\mathrm{AdS}_{3}$ gravity coupled to fields of spin greater than 2}

The extension of our boundary conditions and the analysis of the properties of the corresponding higher spin black flower can also be performed in the case of gravity with negative cosmological constant coupled to bosonic fields of spin $s>2$. As explained in the introduction, the generic theory we consider ${ }^{2}$ is described by a gauge group spanned by two copies of $s l(N, \mathbb{R})$, where the two copies of $s l(2, \mathbb{R})$ are principally embedded. It is also worth mentioning that in the case of even $N$, the theory admits an additional truncation that describes the coupling of gravitation on $\mathrm{AdS}_{3}$ with fields of even spin $s=4,6, \cdots$, $N$, described by two copies of $s p(N, \mathbb{R})$. Additional special truncations are also known to exist in the case of exceptional groups, see e.g., [26]. Hereafter we carry out the analysis for $\operatorname{sl}(N, \mathbb{R})$.

We propose that the asymptotic behaviour of the $s l(N, \mathbb{R})$ gauge fields $A^{ \pm}$is of the form in (2.1) with $b_{ \pm}$given by (2.2), so that the auxiliary connection extends to

$$
a^{ \pm}=\left( \pm \mathcal{J}^{ \pm} d \varphi+\zeta^{ \pm} d t\right) \mathrm{L}_{0}+\sum_{s=3}^{N}\left( \pm \mathcal{J}_{(s)}^{ \pm} d \varphi+\zeta_{(s)}^{ \pm} d t\right) \mathrm{W}_{0}^{(s)}
$$

where $\mathrm{L}_{0}$, and $\mathrm{W}_{0}^{(s)}$ stand for the generators of the Cartan subalgebra of the gauge group. The dynamical fields are then described by arbitrary functions of time and the angular coordinate, given by $\mathcal{J}^{ \pm}, \mathcal{J}_{(s)}^{ \pm}$, with $s=3,4, \cdots, N$; and their corresponding Lagrange multipliers, $\zeta^{ \pm}, \zeta_{(s)}^{ \pm}$, are assumed to be fixed at the boundary without variation. It is then simple to verify that the field equations read

$$
\dot{\mathcal{J}}^{ \pm}= \pm \zeta^{\prime} \quad \dot{\mathcal{J}}_{(s)}^{ \pm}= \pm \zeta_{(s)}^{\prime} .
$$

The asymptotic symmetries that maintain the form of (6.1) are spanned by Lie-algebravalued parameters of the form $\epsilon^{ \pm}=\eta^{ \pm} \mathrm{L}_{0}+\sum_{s=3}^{N} \eta_{(s)}^{ \pm} \mathrm{W}_{0}^{(s)}$, with $\dot{\eta}^{ \pm}=\dot{\eta}_{(s)}^{ \pm}=0$, provided the fields transform according to

$$
\delta \mathcal{J}^{ \pm}= \pm \eta^{ \pm \prime} \quad \delta \mathcal{J}_{(s)}^{ \pm}= \pm \eta_{(s)}^{ \pm \prime}
$$

\footnotetext{
${ }^{2}$ Further generalizations to higher-spin theories based on Chern-Simons actions (1.1) with arbitrary gauge groups $G^{+}, G^{-}$containing SL $(2, \mathbb{R})$ as subgroup are possible as well, but will not be considered in the present work. See for instance section 2.1 in [10].
} 
The canonical generators of the asymptotic symmetries then read

$$
\mathcal{Q}^{ \pm}\left[\eta^{ \pm}, \eta_{(s)}^{ \pm}\right]=\mp \frac{k}{4 \pi} \int d \varphi\left(\eta^{ \pm}(\varphi) \mathcal{J}^{ \pm}(\varphi)+\sum_{s=3}^{N} \alpha_{s} \eta_{(s)}^{ \pm}(\varphi) \mathcal{J}_{(s)}^{ \pm}(\varphi)\right)
$$

where

$$
\alpha_{s}=\frac{48(s-1) !^{4}}{(2 s-1) !(2 s-2) !} \prod_{i=2}^{s-1}\left(N^{2}-i^{2}\right)
$$

and their algebra is given by

$$
\begin{aligned}
\left\{\mathcal{J}^{ \pm}(\varphi), \mathcal{J}^{ \pm}\left(\varphi^{\prime}\right)\right\} & =\mp\left(\frac{4 \pi}{k}\right) \delta^{\prime}\left(\varphi-\varphi^{\prime}\right) \\
\left\{\mathcal{J}_{(s)}^{ \pm}(\varphi), \mathcal{J}_{\left(s^{\prime}\right)}^{ \pm}\left(\varphi^{\prime}\right)\right\} & =\mp\left(\frac{4 \pi}{\alpha_{s} k}\right) \delta_{s, s^{\prime}} \delta^{\prime}\left(\varphi-\varphi^{\prime}\right) .
\end{aligned}
$$

Expanding in Fourier modes according to

$$
\mathcal{J}^{ \pm}(\varphi)=\frac{2}{k} \sum_{n=-\infty}^{\infty} J_{n}^{ \pm} e^{ \pm i n \varphi} \quad \mathcal{J}_{(s)}^{ \pm}(\varphi)=\frac{2}{\alpha_{s} k} \sum_{n=-\infty}^{\infty} J_{n}^{(s) \pm} e^{ \pm i n \varphi}
$$

the algebra (6.6) reads

$$
i\left\{J_{n}^{ \pm}, J_{m}^{ \pm}\right\}=\frac{k}{2} n \delta_{m+n, 0} \quad i\left\{J_{n}^{(s) \pm}, J_{m}^{\left(s^{\prime}\right) \pm}\right\}=\frac{\alpha_{s} k}{2} n \delta_{s, s^{\prime}} \delta_{m+n, 0}
$$

which corresponds to a set of $\hat{u}(1)$ currents with levels $\frac{1}{2} k$ and $\frac{1}{2} \alpha_{s} k$.

For the simplest choice of boundary conditions, given by constant Lagrange multipliers $\zeta^{ \pm}, \zeta_{(s)}^{ \pm}$, the dynamical fields become time-independent, and the total Hamiltonian reads

$$
H=\zeta^{+} J_{0}^{+}+\zeta^{-} J_{0}^{-}+\sum_{s=3}^{N}\left(\zeta_{(s)}^{+} J_{0}^{(s)+}+\zeta_{(s)}^{-} J_{0}^{(s)-}\right)
$$

which manifestly commutes with the $\hat{u}(1)$ generators $J_{n}^{ \pm}, J_{m}^{(s) \pm}$. Therefore, in this case, the whole set of affine global charges can be regarded as (higher spin) soft hair in the sense of $[2]$.

Following the procedure described in section 3 , one naturally expects to find the generalization of the Miura transformation in (3.9), (3.10) between the currents in the diagonal and highest weight gauges, from which the $W_{N}$ currents should emerge from composite operators of the affine ones through an analogue of the twisted Sugawara construction.

Note that in the case of constant $\zeta^{ \pm}$and $\zeta_{(s)}^{ \pm}$, the field equations (6.2) imply that the space of solutions that fulfills our boundary conditions is described by $\mathcal{J}^{ \pm}=\mathcal{J}^{ \pm}(\varphi)$, $\mathcal{J}_{(s)}^{ \pm}=\mathcal{J}_{(s)}^{ \pm}(\varphi)$, which generically corresponds to non spherically symmetric higher spin black holes endowed with all of the possible left and right soft $\hat{u}(1)$ charges. Indeed, in the fundamental representation of $\operatorname{sl}(N, \mathbb{R})$, regularity of the Euclidean configurations reads

$$
\mathcal{H}_{\mathcal{C}}=\mathcal{P} e^{\int_{\mathcal{C}} a}=(-1)^{N+1} \mathbb{1},
$$


where $\mathcal{C}$ stands for any contractible cycle in the solid torus. The regularity conditions of the Euclidean solutions then just reduces to exponentiate a diagonal matrix, which readily implies that the chemical potentials $\zeta^{ \pm}, \zeta_{(s)}^{ \pm}$become fixed by precise relationships that depend linearly on $N-1$ arbitrary integers, and they are independent of the global charges. For the branch that is connected to the BTZ black hole, the only nonvanishing chemical potentials are the ones that correspond to the purely gravitational sector, i.e.,

$$
\zeta^{ \pm}=\frac{2 \pi}{\beta} \quad \zeta_{(s)}^{ \pm}=0 .
$$

The generic solution is then described by $N-1$ left and right soft $\hat{u}(1)$ charges, from which the higher spin ones can be reconstructed by virtue of the map between them and the $W_{N}$ currents.

For the branch that is continuously connected to the BTZ black hole, the entropy of a generic higher spin black flower can be directly recovered from (4.4), which reduces to

$$
S=2 \pi\left(J_{0}^{+}+J_{0}^{-}\right)
$$

Note that the entropy (6.11) exactly agrees with the one in (4.6) for the case of $\operatorname{sl}(3, \mathbb{R})$, as well as for the expression found in [1] in the case of gravity on $\mathrm{AdS}_{3}$. In this sense, the entropy in (6.11) is universal because it depends only on the zero modes of the purely gravitational $\hat{u}(1)$ charges, regardless the value of $N \geq 2$.

\section{Discussion}

The new set of boundary conditions (2.1)-(2.3) (or more generally, (6.1)) for higher spin gravity on $\mathrm{AdS}_{3}$ has a set of affine $\hat{u}(1)$ currents generating the asymptotic symmetry algebra with non-vanishing levels (2.10) (or more generally (6.8)). The concept of spin emerges from the conformal weight of the composite generators of the asymptotic $W$ algebra that is recovered through a nonlinear analogue of the twisted Sugawara construction (3.9), (3.10). Fourier decomposition of the $\hat{u}(1)$ charges leads to a tower of generators (2.9) which can be interpreted as creation operators for negative integer indices. Acting with these creation operators on states, e.g. on the vacuum as in (2.11), generates higher spin soft hair descendants that have the same energy as the original state, thereby generalizing corresponding spin-2 results [2] (see also [27-36]).

The generic solution of the field equations that fulfills our boundary conditions describes stationary and non necessarily spherically symmetric configurations, whose entropy (6.11) was shown to be independent of (higher spin) soft hair. In the case of $\mathrm{AdS}_{3}$ gravity coupled to spin-3 fields, these results were also explicitly recovered in the metric formalism in section 5. It would be interesting to explore further geometric aspects of these higher spin black flowers along the lines of $[37,38]$, as well as the possibility of performing a microstate counting for their entropy as in [39].

We have generalized our results from spin-3 gravity in $\mathrm{AdS}_{3}$ to general higher spin gravity in section 6 , but there is a number of issues that remain for further exploration. For instance, we did not explicitly provide the (twisted) Sugawara-like constructions for 
spins greater than 3. Moreover, we focused exclusively on the principal embedding of $s l(2, \mathbb{R})$ into $s l(N, \mathbb{R})[40,41]$. Additionally, we did not consider Vasiliev-type of theories with infinite towers of spins, based on $h s(\lambda)[7,9]$. Finally, our focus was exclusively on $\mathrm{AdS}_{3}$ backgrounds, but it is well-known that higher spin gravity allows also for non-AdS backgrounds [42-46] and vanishing cosmological constant [47-51] or generalizations along the lines of hypergravity [52-55]. Investigations of the other branches, besides the BTZ branch, of the entropy should be possible [56]. Furthermore, it might be interesting to explore extremal higher spin black holes $[55,57]$ and entanglement entropy [58-62] in this setup.

Besides, a different set of boundary conditions for which the Lagrange multipliers depend locally on the dynamical fields has been recently proposed in [63], where it was shown that the field equations correspond to different integrable hierarchies. Our set of boundary conditions, once expressed in the highest weight gauge, differs from the one in [63] because here the Lagrange multipliers depend non-locally on the dynamical fields (see e.g., eqs. (3.7), (3.8), with (3.9), (3.10)). Nonetheless, as pointed out in [63], for the case of pure gravity on $\mathrm{AdS}_{3}$, our boundary conditions appear to be related to a representative of an extension of the KdV hierarchy that is labeled by a fractional instead of an integer label (precisely $k=-1 / 2$ ), see e.g. [64-67]. One might then naturally expect that a similar effect should occur for the extension of different hierarchies once the higher spin fields within our boundary conditions are switched on.

Additional interesting possibilities also naturally arise along the lines of the connection of higher spin fields and string theory $[68,69]$ and the diverse related results in refs. [70-80].

\section{Acknowledgments}

We thank H. Afshar, G. Barnich, X. Bekaert, A. Campoleoni, D. Francia, O. Fuentealba, M. Gary, J. Matulich, W. Merbis, M. Riegler and J. Simon for useful comments. We also acknowledge the scientific atmosphere of the workshops "Topics in three dimensional Gravity" in March 2016 at the Abdus Salam ICTP, Trieste, Italy, the workshop "Flat Holography" in April 2016 at the Simons Center, Stony Brook, New York and the MIAPP programme "Higher-Spin Theory and Duality" in May 2016 at the Munich Institute for Astro- and Particle Physics, Germany.

The work of AP, DT and RT is partially funded by Fondecyt grants № 11130260 , 11130262, 1130658, 1161311. The Centro de Estudios Científicos (CECs) is funded by the Chilean Government through the Centers of Excellence Base Financing Program of Conicyt. DG was supported by the Austrian Science Fund (FWF) projects P 27182-N27 and P 28751-N27 and by the program Science without Borders, project CNPq-401180/20140. SP was supported by the FWF project P 27396-N27.

\section{A Principal embedding of SL $(2, \mathbb{R})$ within $\mathrm{SL}(N, \mathbb{R})$}

In the principal embedding of SL $(2, \mathbb{R})$ within $\mathrm{SL}(N, \mathbb{R})$, the set of generators of $\operatorname{sl}(N, \mathbb{R})$ naturally splits as $\left\{\mathrm{L}_{i} ; \mathrm{W}_{m}^{(s)}\right\}$, with $i=-1,0,1 ; s=3, \cdots, N$, and $m=-(s-1), \cdots,(s-1)$, 
so that

$$
\begin{aligned}
{\left[\mathrm{L}_{i}, \mathrm{~L}_{j}\right] } & =(i-j) \mathrm{L}_{i+j} \\
{\left[\mathrm{~L}_{i}, \mathrm{~W}_{m}^{(s)}\right] } & =((s-1) i-m) \mathrm{W}_{i+m}^{(s)}
\end{aligned}
$$

from which $\mathrm{L}_{i}$ and $\mathrm{W}_{m}^{(s)}$ can be seen to possess spin two and spin $s$, respectively. In the fundamental representation, the generators of the $s l(2, \mathbb{R})$ subset are described through $N \times N$ matrices that can be chosen to be given by

$$
\begin{aligned}
\left(\mathrm{L}_{1}\right)_{j k} & =-\sqrt{j(N-j)} \delta_{j+1, k} \\
\left(\mathrm{~L}_{-1}\right)_{j k} & =\sqrt{k(N-k)} \delta_{j, k+1} \\
\left(\mathrm{~L}_{0}\right)_{j k} & =\frac{1}{2}(N+1-2 j) \delta_{j, k},
\end{aligned}
$$

with $j, k=2 \ldots, N$, so that the remaining ones become defined as

$$
\begin{aligned}
\mathrm{W}_{m}^{(s)} & =2(-1)^{s-m-1} \frac{(s+m-1) !}{(2 s-2) !} \underbrace{\left[\mathrm{L}_{-1},\left[\mathrm{~L}_{-1}, \cdots\left[\mathrm{L}_{-1}\right.\right.\right.}_{s-m-1 \text { terms }},\left(\mathrm{L}_{1}\right)^{s-1}] \cdots]] \\
& =2(-1)^{s-m-1} \frac{(s+m-1) !}{(2 s-2) !}\left(\operatorname{ad}_{\mathrm{L}_{-1}}\right)^{s-m-1}\left(\mathrm{~L}_{1}\right)^{s-1}
\end{aligned}
$$

where $\operatorname{ad}_{\mathrm{X}}(\mathrm{Y}):=[\mathrm{X}, \mathrm{Y}]$.

The normalization constants used in the text are then given by

$$
\begin{aligned}
\epsilon_{N} & =\operatorname{tr}\left(\mathrm{L}_{0} \mathrm{~L}_{0}\right)=\frac{N\left(N^{2}-1\right)}{12} \\
\alpha_{s} & =\frac{12}{N\left(N^{2}-1\right)} \operatorname{tr}\left(\mathrm{W}_{0}^{(s)} \mathrm{W}_{0}^{(s)}\right)=\frac{48(s-1) !^{4}}{(2 s-1) !(2 s-2) !} \prod_{i=2}^{s-1}\left(N^{2}-i^{2}\right) .
\end{aligned}
$$

\section{B Explicit form of the spin-3 field}

For the particular case of the solution that describes a higher spin black flower, whose metric is given by (5.4), the explicit form of the spin-3 field reads

$$
\begin{aligned}
\Phi= & \left\{a^{-2} \gamma_{(3)} d \rho^{2}+2 \ell\left[(1-2 f(\rho))^{2}\left(a_{(3)} a^{-1} \gamma-\frac{1}{3} \gamma_{(3)}\right)-\frac{4}{3} a^{-1} \gamma_{(3)} \frac{\rho}{\ell} f(\rho)\right] d \rho d v\right. \\
& +\frac{2}{3} a^{-1}\left[(1-2 f(\rho))^{2}\left(\gamma_{(3)} \omega-3 \gamma \omega_{(3)}\right)+4 a^{-1} \gamma_{(3)} \omega \frac{\rho}{\ell} f(\rho)\right] d \rho d \varphi \\
& +\frac{2}{3} a^{-1} \ell \rho f(\rho)\left[(1-2 f(\rho))^{2}\left(\gamma_{(3)}\left(a^{2}+4 a_{(3)}^{2}\right)-6 a a_{(3)} \gamma\right)+4 a \gamma_{(3)} \frac{\rho}{\ell} f(\rho)\right] d t^{2} \\
& +\frac{4}{3} a^{-1} \rho f(\rho)\left[(1-2 f(\rho))^{2}\left(3 \gamma\left(a \omega_{(3)}+a_{(3)} \omega\right)-\gamma_{(3)}\left(a \omega+4 a_{(3)} \omega_{(3)}\right)\right)\right. \\
& \left.+4 \omega \gamma_{(3)} \frac{\rho}{\ell} f(\rho)\right] d v d \varphi+\left[(1-2 f(\rho))^{4} \gamma_{(3)}\left(\gamma^{2}-\frac{4}{3} \gamma_{(3)}^{2}\right)\right.
\end{aligned}
$$




$$
\begin{aligned}
& +\frac{1}{3}(1-2 f(\rho))^{2}\left(2 a^{-1}\left(\gamma_{(3)}\left(\omega^{2}+4 \omega_{(3)}^{2}\right)-6 \gamma \omega_{(3)} \omega\right) \frac{\rho}{\ell} f(\rho)-\gamma_{(3)} \gamma^{2}\right) \\
& \left.\left.+\frac{8}{3} a^{-2} \gamma_{(3)}\left(\frac{\rho}{\ell} f(\rho) \omega^{2}+a \gamma_{(3)}^{2}\right) \frac{\rho}{\ell} f(\rho)+\frac{28}{27} \gamma_{(3)}^{3}\right] d \varphi^{2}\right\} d \varphi .
\end{aligned}
$$

Therefore, close to the horizon, the component $\Phi_{\varphi \varphi \varphi}$ expands as in eq. (5.6).

For a generic choice of Lagrange multipliers, the spacetime geometry can be described in normal coordinates as in eq. (5.7), and the exact expression for the spin-3 field is given by

$$
\begin{aligned}
& \Phi=-\frac{\ell^{3}}{3}\left(\frac{1}{2}\left(\zeta_{(3)}^{+}-\zeta_{(3)}^{-}\right) d t+\frac{\gamma_{(3)}}{\ell} d \varphi\right) d r^{2} \\
& -\frac{\ell^{3}}{6}\left\{-\frac{3}{8}\left(\cosh (4 r)+\frac{1}{3}\right)\left[\frac{4}{3} \zeta_{(3)}^{+} \zeta_{(3)}^{-}\left(\zeta_{(3)}^{+}-\zeta_{(3)}^{-}\right)+\zeta_{(3)}^{+}\left(\zeta^{-}\right)^{2}-\zeta_{(3)}^{-}\left(\zeta^{+}\right)^{2}\right]\right. \\
& \left.+\zeta^{+} \zeta^{-}\left(\zeta_{(3)}^{+}-\zeta_{(3)}^{-}\right) \cosh (2 r)+\frac{2}{9}\left[\left(\zeta_{(3)}^{+}\right)^{3}-\left(\zeta_{(3)}^{-}\right)^{3}\right]-\frac{1}{2}\left[\left(\zeta^{+}\right)^{3}-\left(\zeta^{-}\right)^{3}\right]\right\} d t^{3} \\
& -\frac{1}{6}\left\{\gamma_{(3)}^{3}\left(\cosh (4 r)+\frac{7}{9}\right)+\omega_{(3)}\left(3 \gamma \omega-2 \gamma_{(3)} \omega_{(3)}\right) \sinh ^{2}(2 r)\right. \\
& \left.-3 \gamma_{(3)} \omega^{2} \sinh ^{2}(r)\left(\cosh (2 r)-\frac{1}{3}\right)-3 \gamma_{(3)} \gamma^{2} \cosh ^{2}(r)\left(\cosh (2 r)+\frac{1}{3}\right)\right\} d \varphi^{3} \\
& -\ell\left\{\left(\zeta^{+}+\zeta^{-}\right)\left(\gamma \omega_{(3)} \cosh ^{2}(r)+\frac{1}{6} \gamma_{(3)} \omega(1-3 \cosh (2 r))\right) \sinh ^{2}(r)\right. \\
& +\left(\zeta^{+}-\zeta^{-}\right)\left(\omega_{(3)} \omega \sinh ^{2}(r)-\frac{1}{6} \gamma_{(3)} \gamma(3 \cosh (2 r)+1)\right) \cosh ^{2}(r) \\
& +\frac{1}{4}\left(\zeta_{(3)}^{+}+\zeta_{(3)}^{-}\right)\left(\gamma \omega-\frac{4}{3} \gamma_{(3)} \omega_{(3)}\right) \sinh ^{2}(2 r)-\frac{1}{4}\left(\zeta_{(3)}^{+}-\zeta_{(3)}^{-}\right)\left[\frac{2}{3} \omega_{(3)}^{2} \sinh ^{2}(2 r)\right. \\
& -\gamma_{(3)}^{2}\left(\cosh (4 r)+\frac{7}{9}\right)+\gamma^{2} \cosh ^{2}(r)\left(\cosh (2 r)+\frac{1}{3}\right) \\
& \left.\left.+\omega^{2} \sinh ^{2}(r)\left(\cosh (2 r)-\frac{1}{3}\right)\right]\right\} d t d \varphi^{2}-\frac{\ell^{2}}{2}\left\{\omega_{(3)}\left[\left(\zeta^{+}\right)^{2}-\left(\zeta^{-}\right)^{2}\right] \sinh ^{2}(r) \cosh ^{2}(r)\right. \\
& -\frac{1}{6} \gamma_{(3)}\left[\left(\zeta_{(3)}^{+}+\zeta_{(3)}^{-}\right)^{2} \sinh ^{2}(2 r)-\frac{3}{2}\left(\cosh (4 r)+\frac{7}{9}\right)\left(\zeta_{(3)}^{+}-\zeta_{(3)}^{-}\right)^{2}\right. \\
& \left.+\frac{3}{2}\left(\zeta^{+}+\zeta^{-}\right)^{2} \sinh ^{2}(r)\left(\cosh (2 r)-\frac{1}{3}\right)+\frac{3}{2}\left(\zeta^{+}-\zeta^{-}\right)^{2} \cosh ^{2}(r)\left(\cosh (2 r)+\frac{1}{3}\right)\right] \\
& +3\left(\zeta_{(3)}^{+}-\zeta_{(3)}^{-}\right)\left[\left(\zeta^{+}-\zeta^{-}\right) \gamma\left(\cosh (2 r)+\frac{1}{3}\right) \cosh ^{2}(r)+\left(\zeta^{+}+\zeta^{-}\right) \omega\left(\cosh (2 r)-\frac{1}{3}\right) \sinh ^{2}(r)\right] \\
& \left.-\frac{3}{2}\left(\zeta_{(3)}^{+}+\zeta_{(3)}^{-}\right)\left[\left(\zeta^{+}+\zeta^{-}\right) \gamma+\left(\zeta^{+}-\zeta^{-}\right) \omega-\frac{4}{3}\left(\zeta_{(3)}^{+}-\zeta_{(3)}^{-}\right) \omega_{(3)}\right] \sinh ^{2}(2 r)\right\} d \varphi d t^{2}
\end{aligned}
$$

so that the component $\Phi_{\varphi \varphi \varphi}$ expands according to (5.6), with $\mathcal{O}(\rho)$ replaced by $\mathcal{O}\left(r^{2}\right)$, near the horizon. 
Open Access. This article is distributed under the terms of the Creative Commons Attribution License (CC-BY 4.0), which permits any use, distribution and reproduction in any medium, provided the original author(s) and source are credited.

\section{References}

[1] H. Afshar et al., Soft Heisenberg hair on black holes in three dimensions, Phys. Rev. D 93 (2016) 101503 [arXiv:1603.04824] [INSPIRE].

[2] S.W. Hawking, M.J. Perry and A. Strominger, Soft Hair on Black Holes, Phys. Rev. Lett. 116 (2016) 231301 [arXiv:1601.00921] [INSPIRE].

[3] J.D. Brown and M. Henneaux, Central Charges in the Canonical Realization of Asymptotic Symmetries: An Example from Three-Dimensional Gravity, Commun. Math. Phys. 104 (1986) 207 [INSPIRE].

[4] M.P. Blencowe, A Consistent Interacting Massless Higher Spin Field Theory in D $=(2+1)$, Class. Quant. Grav. 6 (1989) 443 [INSPIRE].

[5] E. Bergshoeff, M.P. Blencowe and K.S. Stelle, Area Preserving Diffeomorphisms and Higher Spin Algebra, Commun. Math. Phys. 128 (1990) 213 [INSPIRE].

[6] M.A. Vasiliev, Higher spin gauge theories in four-dimensions, three-dimensions and two-dimensions, Int. J. Mod. Phys. D 5 (1996) 763 [hep-th/9611024] [InSPIRE].

[7] M. Henneaux and S.-J. Rey, Nonlinear $W_{\infty}$ as Asymptotic Symmetry of Three-Dimensional Higher Spin Anti-de Sitter Gravity, JHEP 12 (2010) 007 [arXiv: 1008.4579] [INSPIRE].

[8] A. Campoleoni, S. Fredenhagen, S. Pfenninger and S. Theisen, Asymptotic symmetries of three-dimensional gravity coupled to higher-spin fields, JHEP 11 (2010) 007 [arXiv: 1008.4744] [INSPIRE].

[9] M.R. Gaberdiel and T. Hartman, Symmetries of Holographic Minimal Models, JHEP 05 (2011) 031 [arXiv:1101.2910] [INSPIRE].

[10] A. Campoleoni, S. Fredenhagen and S. Pfenninger, Asymptotic W-symmetries in three-dimensional higher-spin gauge theories, JHEP 09 (2011) 113 [arXiv:1107.0290] [INSPIRE].

[11] A. Campoleoni and M. Henneaux, Asymptotic symmetries of three-dimensional higher-spin gravity: the metric approach, JHEP 03 (2015) 143 [arXiv: 1412.6774] [INSPIRE].

[12] M. Gutperle and P. Kraus, Higher Spin Black Holes, JHEP 05 (2011) 022 [arXiv:1103.4304] [INSPIRE].

[13] A. Castro, E. Hijano, A. Lepage-Jutier and A. Maloney, Black Holes and Singularity Resolution in Higher Spin Gravity, JHEP 01 (2012) 031 [arXiv:1110.4117] [INSPIRE].

[14] M. Henneaux, A. Pérez, D. Tempo and R. Troncoso, Chemical potentials in three-dimensional higher spin anti-de Sitter gravity, JHEP 12 (2013) 048 [arXiv:1309.4362] [INSPIRE].

[15] C. Bunster, M. Henneaux, A. Pérez, D. Tempo and R. Troncoso, Generalized Black Holes in Three-dimensional Spacetime, JHEP 05 (2014) 031 [arXiv: 1404.3305] [INSPIRE].

[16] M. Ammon, M. Gutperle, P. Kraus and E. Perlmutter, Spacetime Geometry in Higher Spin Gravity, JHEP 10 (2011) 053 [arXiv:1106.4788] [INSPIRE]. 
[17] T. Regge and C. Teitelboim, Role of Surface Integrals in the Hamiltonian Formulation of General Relativity, Annals Phys. 88 (1974) 286 [INSPIRE].

[18] P. Bouwknegt and K. Schoutens, W symmetry in conformal field theory, Phys. Rept. 223 (1993) 183 [hep-th/9210010] [INSPIRE].

[19] A. Pérez, D. Tempo and R. Troncoso, Higher spin gravity in 3D: Black holes, global charges and thermodynamics, Phys. Lett. B 726 (2013) 444 [arXiv:1207.2844] [inSPIRE].

[20] A. Pérez, D. Tempo and R. Troncoso, Higher spin black hole entropy in three dimensions, JHEP 04 (2013) 143 [arXiv: 1301.0847] [INSPIRE].

[21] J. de Boer and J.I. Jottar, Thermodynamics of higher spin black holes in AdS $S_{3}$, JHEP 01 (2014) 023 [arXiv: 1302.0816] [INSPIRE].

[22] M. Bañados, C. Teitelboim and J. Zanelli, The Black hole in three-dimensional space-time, Phys. Rev. Lett. 69 (1992) 1849 [hep-th/9204099] [InSPIRE].

[23] M. Bañados, M. Henneaux, C. Teitelboim and J. Zanelli, Geometry of the $(2+1)$ black hole, Phys. Rev. D 48 (1993) 1506 [Erratum ibid. D 88 (2013) 069902] [gr-qc/9302012] [INSPIRE].

[24] A. Campoleoni, S. Fredenhagen, S. Pfenninger and S. Theisen, Towards metric-like higher-spin gauge theories in three dimensions, J. Phys. A 46 (2013) 214017 [arXiv: 1208.1851] [INSPIRE].

[25] M. Bañados, R. Canto and S. Theisen, Higher spin black holes in three dimensions: Remarks on asymptotics and regularity, Phys. Rev. D 94 (2016) 024002 [arXiv:1601.05827] [INSPIRE].

[26] B. Chen, J. Long and Y.-n. Wang, Black holes in Truncated Higher Spin AdS $S_{3}$ Gravity, JHEP 12 (2012) 052 [arXiv: 1209.6185] [INSPIRE].

[27] T. Banks, The Super BMS Algebra, Scattering and Holography, arXiv:1403.3420 [INSPIRE].

[28] G. Dvali, C. Gomez and D. Lüst, Classical Limit of Black Hole Quantum N-Portrait and BMS Symmetry, Phys. Lett. B 753 (2016) 173 [arXiv:1509.02114] [INSPIRE].

[29] L. Donnay, G. Giribet, H.A. Gonzalez and M. Pino, Supertranslations and Superrotations at the Black Hole Horizon, Phys. Rev. Lett. 116 (2016) 091101 [arXiv:1511. 08687] [InSPIRE].

[30] M. Blau and M. O'Loughlin, Horizon Shells and BMS-like Soldering Transformations, JHEP 03 (2016) 029 [arXiv: 1512.02858] [INSPIRE].

[31] A. Averin, G. Dvali, C. Gomez and D. Lüst, Gravitational Black Hole Hair from Event Horizon Supertranslations, JHEP 06 (2016) 088 [arXiv:1601.03725] [INSPIRE].

[32] A. Kehagias and A. Riotto, BMS in Cosmology, JCAP 05 (2016) 059 [arXiv:1602.02653] [INSPIRE].

[33] C. Eling and Y. Oz, On the Membrane Paradigm and Spontaneous Breaking of Horizon BMS Symmetries, JHEP 07 (2016) 065 [arXiv:1605.00183] [INSPIRE].

[34] M.R. Setare and H. Adami, Near Horizon Symmetries of the Non-Extremal Black Hole Solutions of Generalized Minimal Massive Gravity, Phys. Lett. B 760 (2016) 411 [arXiv: 1606.02273] [INSPIRE].

[35] M.R. Setare and H. Adami, The Heisenberg algebra as near horizon symmetry of the black flower solutions of Chern-Simons-like theories of gravity, arXiv:1606.05260 [INSPIRE]. 
[36] A. Averin, G. Dvali, C. Gomez and D. Lüst, Goldstone origin of black hole hair from supertranslations and criticality, arXiv:1606.06260 [INSPIRE].

[37] G. Barnich, C. Troessaert, D. Tempo and R. Troncoso, Asymptotically locally flat spacetimes and dynamical nonspherically-symmetric black holes in three dimensions, Phys. Rev. D 93 (2016) 084001 [arXiv: 1512.05410] [INSPIRE].

[38] C. Troessaert, D. Tempo and R. Troncoso, Asymptotically flat black holes and gravitational waves in three-dimensional massive gravity, in proceedings of 8th Aegean Summer School: Gravitational Waves: From Theory to Observations, Rethymno, Crete, Greece, June 29-July 042015 [arXiv: 1512.09046] [INSPIRE].

[39] H. Afshar, D. Grumiller and M.M. Sheikh-Jabbari, Near Horizon Soft Hairs as Microstates of Three Dimensional Black Holes, arXiv:1607.00009 [INSPIRE].

[40] A. Castro, E. Hijano and A. Lepage-Jutier, Unitarity Bounds in AdS $S_{3}$ Higher Spin Gravity, JHEP 06 (2012) 001 [arXiv: 1202.4467] [INSPIRE].

[41] H. Afshar, M. Gary, D. Grumiller, R. Rashkov and M. Riegler, Semi-classical unitarity in 3-dimensional higher-spin gravity for non-principal embeddings, Class. Quant. Grav. 30 (2013) 104004 [arXiv:1211.4454] [INSPIRE].

[42] M. Gary, D. Grumiller and R. Rashkov, Towards non-AdS holography in 3-dimensional higher spin gravity, JHEP 03 (2012) 022 [arXiv:1201.0013] [INSPIRE].

[43] C. Krishnan, A. Raju, S. Roy and S. Thakur, Higher Spin Cosmology, Phys. Rev. D 89 (2014) 045007 [arXiv: 1308.6741] [INSPIRE].

[44] M. Gutperle, E. Hijano and J. Samani, Lifshitz black holes in higher spin gravity, JHEP 04 (2014) 020 [arXiv:1310.0837] [INSPIRE].

[45] M. Gary, D. Grumiller, S. Prohazka and S.-J. Rey, Lifshitz Holography with Isotropic Scale Invariance, JHEP 08 (2014) 001 [arXiv:1406.1468] [INSPIRE].

[46] V. Breunhölder, M. Gary, D. Grumiller and S. Prohazka, Null warped AdS in higher spin gravity, JHEP 12 (2015) 021 [arXiv:1509.08487] [INSPIRE].

[47] H. Afshar, A. Bagchi, R. Fareghbal, D. Grumiller and J. Rosseel, Spin-3 Gravity in Three-Dimensional Flat Space, Phys. Rev. Lett. 111 (2013) 121603 [arXiv:1307.4768] [INSPIRE].

[48] H.A. Gonzalez, J. Matulich, M. Pino and R. Troncoso, Asymptotically flat spacetimes in three-dimensional higher spin gravity, JHEP 09 (2013) 016 [arXiv:1307.5651] [INSPIRE].

[49] M. Gary, D. Grumiller, M. Riegler and J. Rosseel, Flat space (higher spin) gravity with chemical potentials, JHEP 01 (2015) 152 [arXiv:1411.3728] [INSPIRE].

[50] J. Matulich, A. Pérez, D. Tempo and R. Troncoso, Higher spin extension of cosmological spacetimes in 3D: asymptotically flat behaviour with chemical potentials and thermodynamics, JHEP 05 (2015) 025 [arXiv: 1412.1464] [INSPIRE].

[51] H. Afshar et al., Soft hairy horizons in three spacetime dimensions, CECS-PHY-16/05.

[52] O. Fuentealba, J. Matulich and R. Troncoso, Asymptotically flat structure of hypergravity in three spacetime dimensions, JHEP 10 (2015) 009 [arXiv: 1508.04663] [INSPIRE].

[53] M. Henneaux, A. Pérez, D. Tempo and R. Troncoso, Extended anti-de Sitter Hypergravity in $2+1$ Dimensions and Hypersymmetry Bounds, arXiv:1512.08603 [INSPIRE]. 
[54] O. Fuentealba, J. Matulich and R. Troncoso, Extension of the Poincaré group with half-integer spin generators: hypergravity and beyond, JHEP 09 (2015) 003 [arXiv: 1505.06173] [INSPIRE].

[55] M. Henneaux, A. Pérez, D. Tempo and R. Troncoso, Hypersymmetry bounds and three-dimensional higher-spin black holes, JHEP 08 (2015) 021 [arXiv:1506.01847] [INSPIRE].

[56] J.R. David, M. Ferlaino and S.P. Kumar, Thermodynamics of higher spin black holes in 3D, JHEP 11 (2012) 135 [arXiv:1210.0284] [INSPIRE].

[57] M. Bañados, A. Castro, A. Faraggi and J.I. Jottar, Extremal Higher Spin Black Holes, JHEP 04 (2016) 077 [arXiv: 1512.00073] [INSPIRE].

[58] M. Ammon, A. Castro and N. Iqbal, Wilson Lines and Entanglement Entropy in Higher Spin Gravity, JHEP 10 (2013) 110 [arXiv:1306.4338] [INSPIRE].

[59] J. de Boer and J.I. Jottar, Entanglement Entropy and Higher Spin Holography in AdS $S_{3}$, JHEP 04 (2014) 089 [arXiv: 1306.4347] [INSPIRE].

[60] S. Datta, J.R. David, M. Ferlaino and S.P. Kumar, Higher spin entanglement entropy from CFT, JHEP 06 (2014) 096 [arXiv: 1402.0007] [InSPIRE].

[61] A. Castro and E. Llabrés, Unravelling Holographic Entanglement Entropy in Higher Spin Theories, JHEP 03 (2015) 124 [arXiv:1410.2870] [INSPIRE].

[62] J. de Boer, A. Castro, E. Hijano, J.I. Jottar and P. Kraus, Higher spin entanglement and $\mathcal{W}_{\mathrm{N}}$ conformal blocks, JHEP 07 (2015) 168 [arXiv:1412.7520] [INSPIRE].

[63] A. Pérez, D. Tempo and R. Troncoso, Boundary conditions for General Relativity on $A d S_{3}$ and the KdV hierarchy, JHEP 06 (2016) 103 [arXiv: 1605.04490] [INSPIRE].

[64] R.S. Johnson, A non-linear equation incorporating damping and dispersion, J. Fluid Mech. 42 (1970) 49.

[65] Z. Feng, On explicit exact solutions to the compound Burgers-KdV equation, Phys. Lett. A 293 (2002) 57.

[66] Q. Wang, Numerical solutions for fractional KdV-Burgers equation by Adomian decomposition method, Appl. Math. Comput. 182 (2006) 1048.

[67] M. Younis, Soliton solutions of fractional order KdV-Burgers's equation, J. Adv. Phys. 3 (2014) 325.

[68] M.R. Gaberdiel and R. Gopakumar, Higher Spins 83 Strings, JHEP 11 (2014) 044 [arXiv: 1406.6103] [INSPIRE].

[69] M.R. Gaberdiel and R. Gopakumar, String Theory as a Higher Spin Theory, JHEP 09 (2016) 085 [arXiv: 1512.07237] [INSPIRE].

[70] H.-S. Tan, Aspects of Three-dimensional Spin-4 Gravity, JHEP 02 (2012) 035 [arXiv:1111.2834] [INSPIRE].

[71] M. Ferlaino, T. Hollowood and S.P. Kumar, Asymptotic symmetries and thermodynamics of higher spin black holes in $A d S_{3}$, Phys. Rev. D 88 (2013) 066010 [arXiv:1305.2011] [INSPIRE].

[72] G. Compère and W. Song, $\mathcal{W}$ symmetry and integrability of higher spin black holes, JHEP 09 (2013) 144 [arXiv: 1306.0014] [INSPIRE]. 
[73] G. Compère, J.I. Jottar and W. Song, Observables and Microscopic Entropy of Higher Spin Black Holes, JHEP 11 (2013) 054 [arXiv: 1308.2175] [INSPIRE].

[74] W. Li, F.-L. Lin and C.-W. Wang, Modular Properties of 3D Higher Spin Theory, JHEP 12 (2013) 094 [arXiv: 1308.2959] [INSPIRE].

[75] J. de Boer and J.I. Jottar, Boundary conditions and partition functions in higher spin $A d S_{3} / C F T_{2}, J H E P 04$ (2016) 107 [arXiv:1407.3844] [INSPIRE].

[76] A. Cabo-Bizet, E. Gava, V.I. Giraldo-Rivera and K.S. Narain, Black Holes in the 3D Higher Spin Theory and Their Quasi Normal Modes, JHEP 11 (2014) 013 [arXiv:1407.5203] [INSPIRE].

[77] A. Cabo-Bizet and V.I. Giraldo-Rivera, About the phase space of SL(3) Black Holes, JHEP 03 (2015) 081 [arXiv: 1407.8241] [INSPIRE].

[78] W. Li and S. Theisen, Some aspects of holographic W-gravity, JHEP 08 (2015) 035 [arXiv: 1504.07799] [INSPIRE].

[79] L. Apolo, The covariant action of higher spin black holes in three dimensions, JHEP 05 (2016) 097 [arXiv : 1511.01406] [INSPIRE].

[80] L. Apolo and B. Sundborg, The Sky is the Limit: Free Boundary Conditions in $A_{d} S_{3}$ Chern-Simons Theory, in proceedings of the International Workshop on Higher Spin Gauge Theories, Singapore, November 04-06 2015 [arXiv: 1603.03213] [INSPIRE]. 